Check for updates

Cite this: J. Mater. Chem. B, 2021, 9, 7007

Received 1st April 2021,

Accepted 6th May 2021

DOI: $10.1039 / d 1 t b 00725 d$

rsc.li/materials-b

\section{Biomacromolecule-based photo-thermal agents for tumor treatment}

\author{
Bin Liu, $\uparrow^{\mathrm{ab}}$ Fuquan Jiang, $\uparrow^{\mathrm{a}}$ Jing Sun, (D) *c Fan Wang ${ }^{(\mathbb{D}} *^{\mathrm{b}}$ and Kai Liu*bd
}

Cancer treatment has become one of the biggest challenges in modern medicine. Recently, many efforts have been devoted to treat tumors by surgical resection, radiotherapy, or chemotherapy. In comparison to these methods, photo-thermal therapy (PTT) with noninvasive, controllable, direct, and precise characteristics has received tremendous attention in eliminating tumor cells over the past decades. In particular, PTT based on biomacromolecule-based photo-thermal agents (PTAs) outperforms other systems with high photo-thermal efficiency, simple coating, and low immunogenicity. Considering the unique advantages of biomacromolecule-based PTAs in tumor treatment, it is necessary to summarize the recent progress in the field of biomacromolecule-based PTAs for tumor treatment. Herein, this minireview outlines recent progress in the fabrication and applications of biomacromolecule-based PTAs. Within this framework, various types of biomacromolecule-based PTAs are highlighted, including cell-based agents, protein-based agents, nucleotide-based agents, and polysaccharide-based PTAs. In each section, the functional design, photo-thermal effects, and potential clinical applications of each type of PTA are discussed. Finally, a brief perspective for the development of biomacromolecule-based PTAs is presented.

\section{Introduction}

Malignant tumors are considered as a dominant cause of human death and disability. ${ }^{1-3}$ The traditional clinical treatments for

\footnotetext{
${ }^{a}$ Department of Urology, China-Japan Union Hospital of Jilin University, Changchun 130033, China

${ }^{b}$ State Key Laboratory of Rare Earth Resource Utilization, Changchun Institute of Applied Chemistry, Chinese Academy of Sciences, Changchun 130022, China

${ }^{c}$ Institute of Organic Chemistry, University of Ulm, Albert-Einstein-Allee 11, 89081, Ulm, Germany

${ }^{d}$ Department of Chemistry, Tsinghua University, Beijing 100084, China

$\dagger$ These authors contributed equally to this work.
}

tumors mainly include surgical resection, ${ }^{4}$ radiation therapy, ${ }^{5}$ and chemotherapy. ${ }^{6,7}$ However, these methods do not always eliminate cancer cells completely and are often accompanied by a series of related complications. ${ }^{8,9}$ Recently, photo-thermal therapy (PTT) has emerged as a promising cancer treatment relying on the thermal necrosis/apoptosis of cancer cells by specific laser irradiation. ${ }^{10-12}$ As a consequence, PTT has attracted great attention owing to its high therapeutic efficiency, minimal invasiveness, and great penetration depth when compared to traditional treatments. ${ }^{13,14}$ Over the past decades, various near infrared (NIR) light-triggered photo-thermal agents (PTAs) have been widely investigated in cancer treatment, such as organic

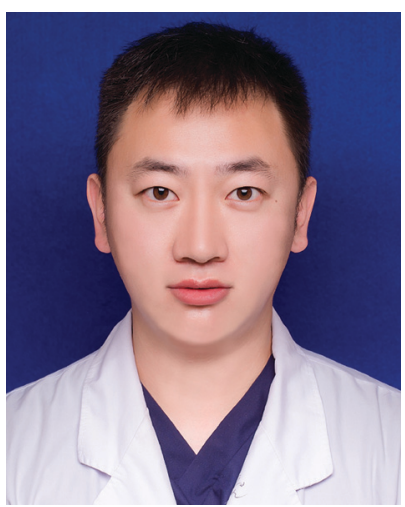

Bin Liu
Bin Liu received his master's degree in medicine from Jilin University in 2015 and then has worked as a surgeon in the Department of Urology to date. From 2018 to 2021, he did his doctorate in Changchun Institute of Applied Chemistry for joint training. His current research topics involve the synthesis of photo-responsive nanomaterials and anti-tumor applications in urothelial carcinoma.

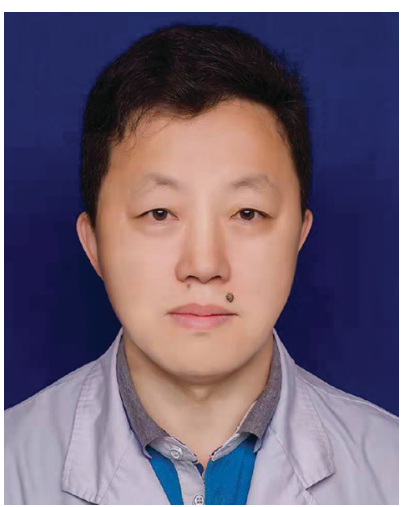

Fuquan Jiang received his PhD degree in urology from Jilin University in 2019 under the supervision of Prof. Gu. He is currently a deputy chief doctor in China-Japan Union Hospital of Jilin University. His research interests focus on the diagnosis and treatment of genitourinary tumors.
Fuquan Jiang 
and inorganic nanomaterials. ${ }^{15,16}$ A large number of inorganicbased PTAs, such as noble metal nanomaterials (gold, ${ }^{17}$ platinum and silver ${ }^{18}$ ), carbon nanomaterials, ${ }^{19}$ quantum dots (QDs), ${ }^{20}$ black phosphorus, ${ }^{21}$ and sulfide nanoparticles, have been extensively investigated for cancer treatment in vivo and in vitro. Despite the high photo-thermal effect of these PTAs, their practical clinical applications are still restricted by their weak biodegradability and long-term toxicity concerns. ${ }^{22-24}$ Similarly, biocompatible organic-based PTAs have also been fabricated and used for photo-thermal tumor elimination, such as indocyanine green (ICG), croconine, porphyrin liposomes, and Prussian blue. ${ }^{25}$ However, the photo-stability and low reproducibility of such PTAs lead to low photo-thermal conversion efficiency and tumor uptake efficiency in vivo, ${ }^{26}$ thereby limiting their further biomedical applications. ${ }^{27,28}$ Therefore, it is of great importance to develop alternatives with high photothermal efficiency, good biodegradability and low toxicity.

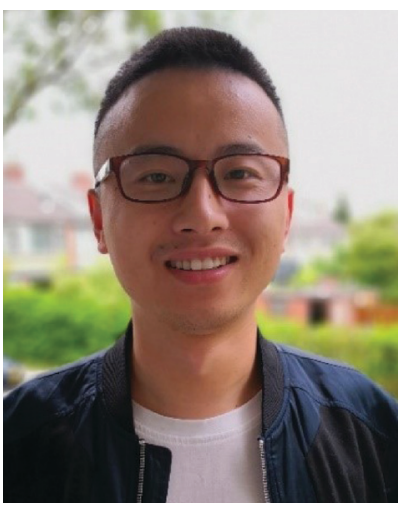

Jing Sun
Jing Sun received his BS degree in chemistry from Southwest University (China) and his MS degree in organic chemistry from Nankai University (China) in 2011 and 2014, respectively. $\mathrm{He}$ obtained his PhD degree from the University of Groningen (The Netherlands) under the supervision of Prof. Andreas Herrmann in 2020, working on engineered protein-based biomaterials. Currently, he is a postdoctoral researcher at the University of Ulm, Germany, focusing on dissipative self-assembly.
Biomacromolecules including cell membrane structures, proteins, nucleic acids, and polysaccharides are common materials for preparing biomimetic nanomaterials. ${ }^{29,30}$ With their specific molecular structures, ${ }^{31}$ innate biocompatibility, and multifunctional properties, ${ }^{32}$ these materials are widely used in drug delivery, ${ }^{33-35}$ bioimaging, ${ }^{36}$ bioengineering, ${ }^{37,38}$ and synthesis of hybrid PTAs. In particular, biomacromolecules like protein and double-stranded DNA can be utilized as templates to synthesize PTAs with specific 3D structures and diameters, exhibiting morphology dependent enhanced tissue penetration and cellular uptake. ${ }^{39,40}$ The highly hydrophilic nature of biomacromolecule-based PTAs allows them to be used under physiological conditions. Additionally, owing to their intrinsic nature, biomacromolecule-based PTAs demonstrate excellent biocompatibility and biodegradability, reducing the cytotoxicity of pristine photo-sensitive agents and prolonging their circulation time in vivo, which are beneficial for PTT. The functional domains in antibodies and aptamers further endow the hybrid PTAs with highly specific recognition and combination capability towards the corresponding antigens in tumor cells, achieving an active targeting ability. ${ }^{41,42}$ Therefore, biomacromolecule-based PTAs are promising materials to break the current limitations and cause a great revolution for the cancer clinical treatment by integrating diagnosis and PTT with minimized side effects.

Herein, we will summarize recent progress in the fabrication and application of PTAs based on biomacromolecules, such as cytomembranes, proteins, nucleic acids, and polysaccharides. We will highlight four different types of biomacromolecule-based PTAs, including cell-based PTAs, protein-based PTAs, nucleic acidbased PTAs, and polysaccharide-based PTAs (Fig. 1). The sources of cells, effective components, types of photosensitive agents, merits and shortcomings are summarized in Table 1. The key aspects related to the functional design, structural control, and

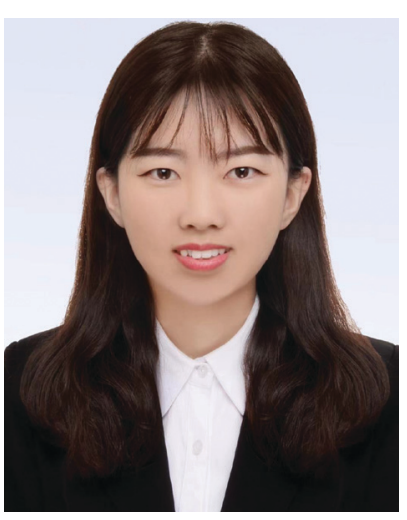

Fan Wang
Fan Wang did her bachelor's study in Lanzhou University in 2012 and then she received her $\mathrm{PhD}$ degree in inorganic chemistry from the University of Chinese Academy of Sciences in China in 2017. From 2017 to 2019, she worked as a research assistant professor at Changchun Institute of Applied Chemistry. In 2020, she was promoted as a research associate professor and now she is focusing on synthesis and biomedical applications of bio-inorganic composite materials.

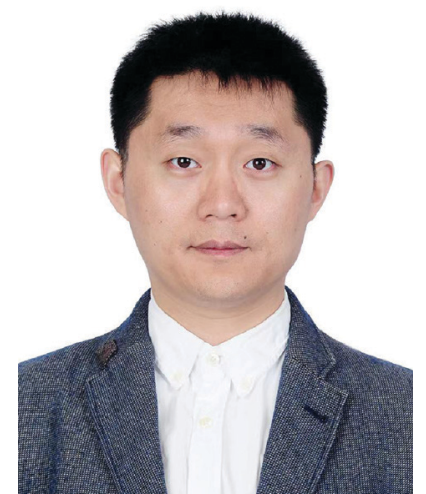

Kai Liu
Kai Liu received his $P h D$ degree from the University of Groningen in The Netherlands in 2015. Then he pursued his postdoctoral studies at the University of Groningen in The Netherlands and Harvard University in the USA, respectively. In 2017 he was appointed as a principal investigator at Changchun Institute of Applied Chemistry of the Chinese Academy of Sciences and focused on engineered biomaterials and high-tech

applications. In 2020, he moved to the Department of Chemistry of Tsinghua University in Beijing, and was promoted as a tenured professor and heads a laboratory performing research on the interface of biotechnology, biosynthetic materials, and information technology. 


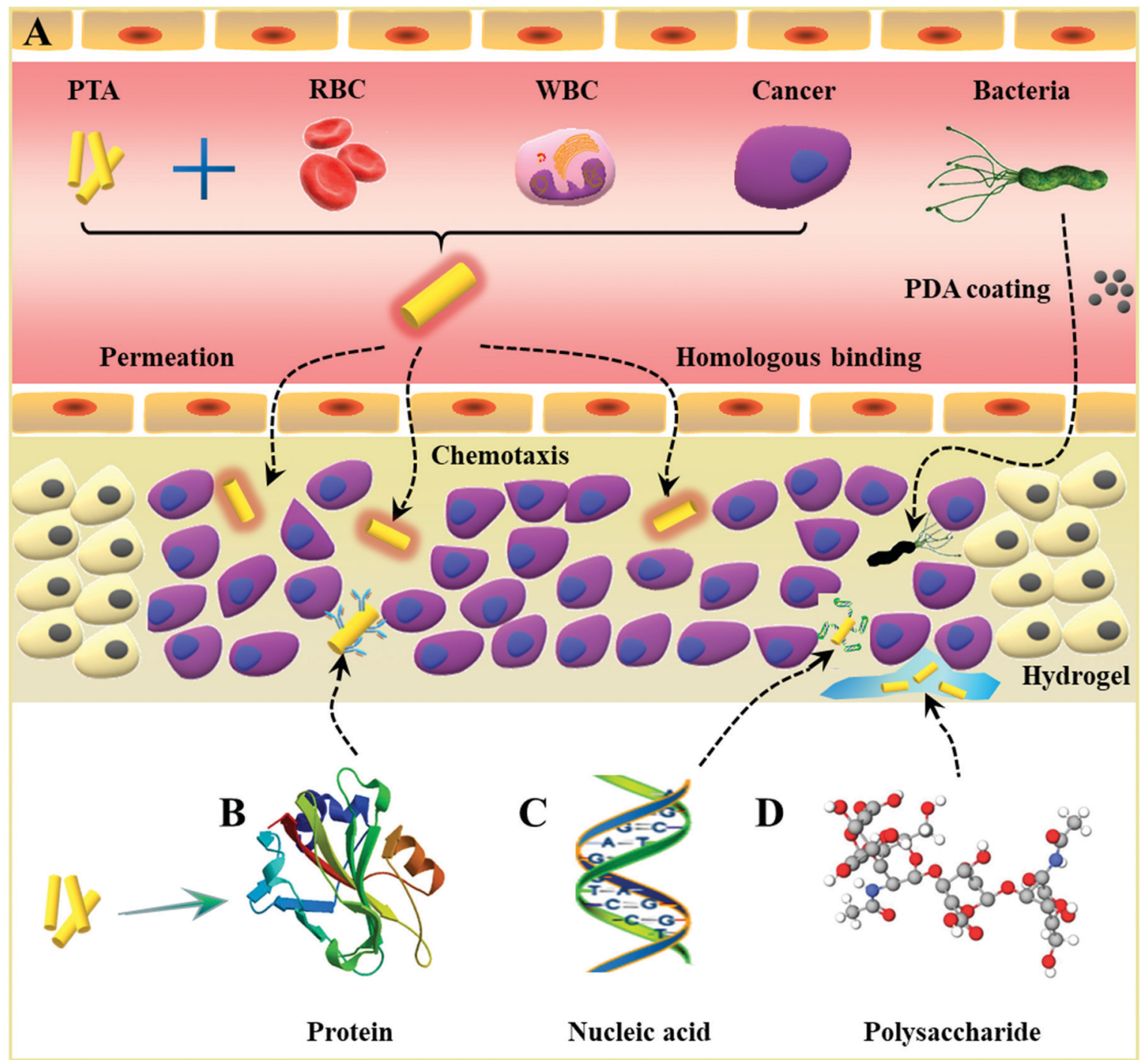

Fig. 1 Schematic illustration of the preparation of multifunctional biological macromolecule based PTAs, e.g., (A) cell-based PTAs, (B) protein-based PTAs, (C) nucleotide-based PTAs and (D) polysaccharide-based PTAs. The improved biomimetic PTAs with good biocompatibility, active tumor targeting, and higher photo-thermal conversion efficiency will be an ideal platform for tumor PTT.

photo-thermal enhancement of each type of biomacromoleculebased PTAs will be discussed in detail. Perspectives and potential challenges toward clinical application with biomacromoleculebased PTAs will also be presented.

\section{Cell-based PTAs}

\subsection{Erythrocyte-based PTAs}

In general, homologous proteins and polysaccharides on the surfaces of red blood cell (RBC) membranes protect them from the attack of immune cells. ${ }^{55}$ Thus, the coating of the cell membrane can reduce the immunogenicity of PTAs and prolong their circulation time. Additionally, red blood cells have no nuclei and organelles. Simple steps such as swelling, ultrasound treatment, and centrifugation can be used to obtain high purity cell membranes. ${ }^{56}$ However, it is difficult to meet the drug concentration required for treatment simply by relying on the enhanced permeability and retention (EPR) effect. Improved efficacies have been obtained by a reasonable construction of erythrocyte-based agents and preventing the loss of generated heat as described below.

Qin et al. successfully explored a new platform based on red blood cell membrane camouflaged melanin (Melanin@RBC) nanoparticles for in vivo antitumor PTT study. ${ }^{43}$ Melanin@RBC nanoparticles were easily produced by mixing and repeatedly extruding (Fig. 2A). Both melanin and RBC membranes were extracted from natural living organisms, resulting in the good biocompatibility and long residence time of the as-obtained nanoparticles. In addition, the obtained Melanin@RBC NPs with strong absorption in the NIR region showed a higher photo-thermal conversion efficiency (40\%) in comparison with melanin-like polydopamine NPs (29\%) (Fig. 2B). With the remarkably higher photo-thermal 
Table 1 Summary of the sources of cells, effective components, photosensitive agents, merits and shortcomings in the application in tumor PTT

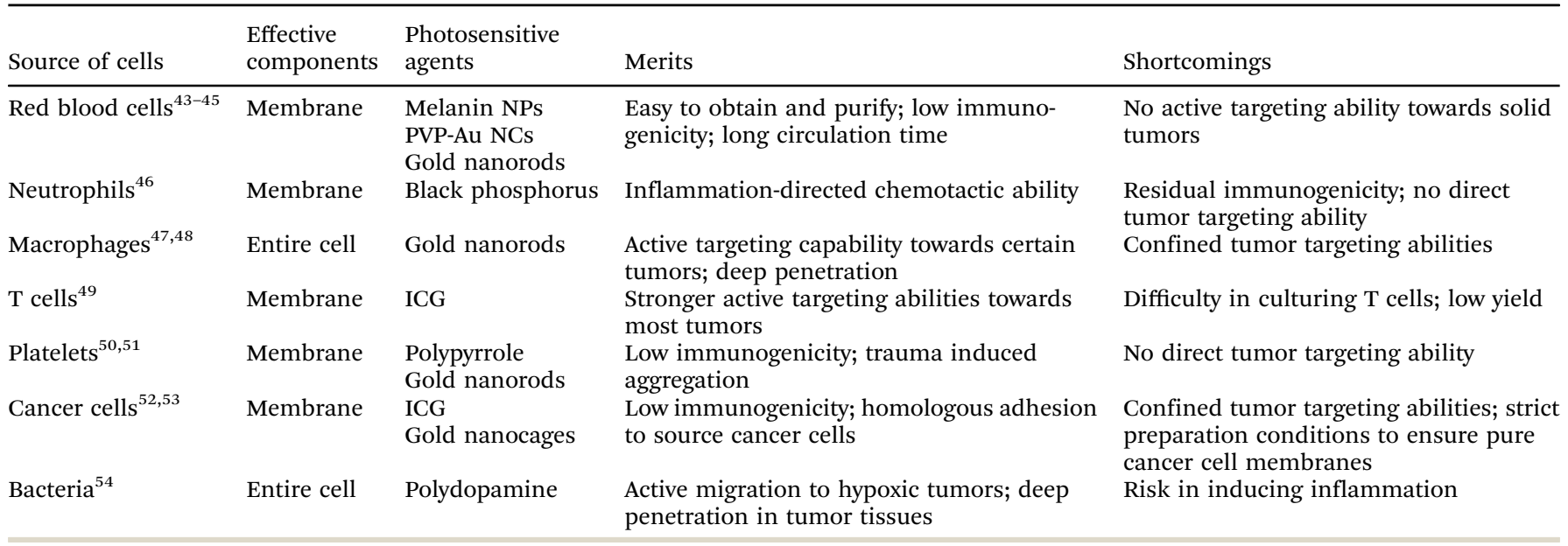

conversion efficiency and optimal accumulation of PTAs at tumors, significant PTT efficacy was achieved in A549 tumor-bearing mice (Fig. 2C).
Piao et al. demonstrated that the coating of the erythrocyte membrane could hinder the diffusion of heat that originated from irradiation, resulting in an influence on the
A

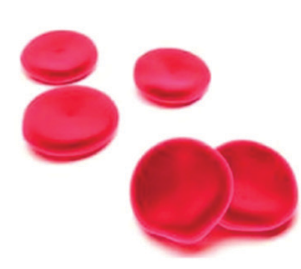

RBCs

B

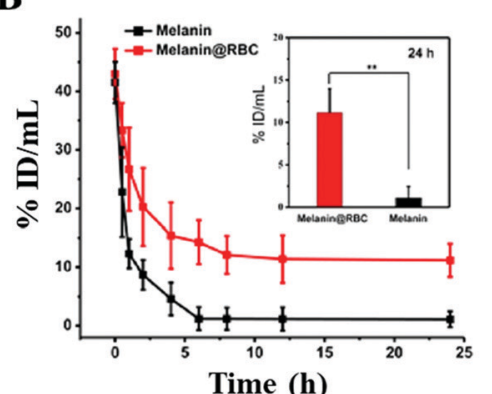

D

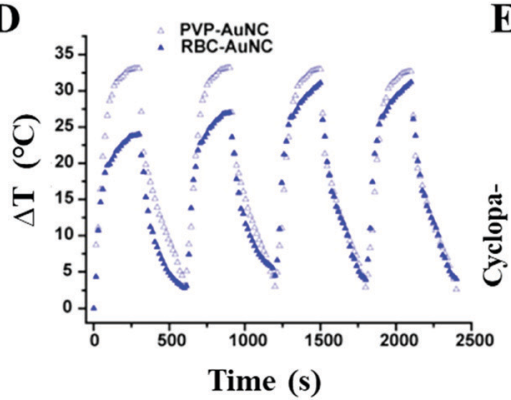

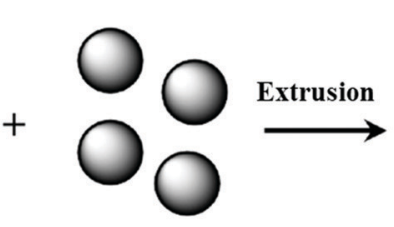

Melanin

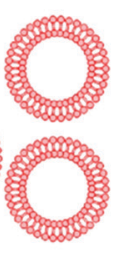

RBC-membrane vesicles

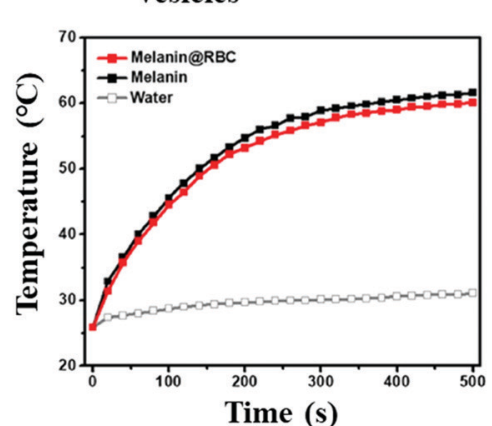

E

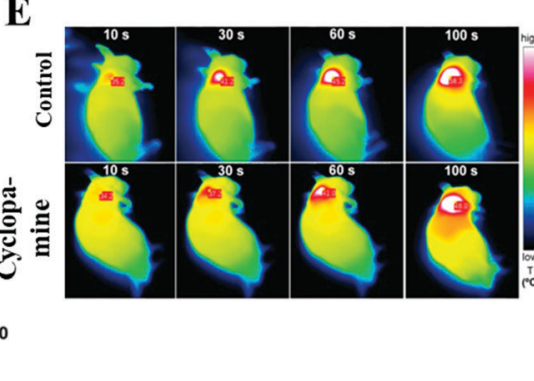

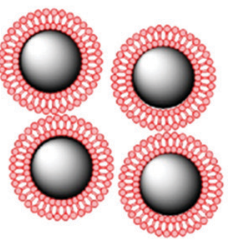

Melanin@RBC
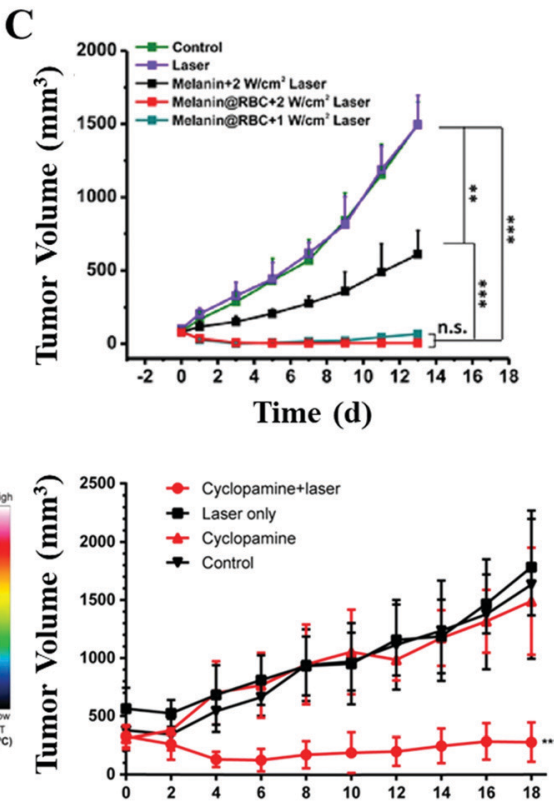

Time (d)

Fig. 2 (A) Schematic illustration of the preparation of RBC membrane camouflaged melanin (Melanin@RBC) nanoparticles. (B) Pharmacokinetics and photo-thermal conversion curves of melanin NPs/MelaninaRBC NPs. (C) Tumor volume curves of A549 tumors in different treatment groups. Reprinted with permission from ref. 43. Copyright 2017, Elsevier. (D) As the extracellular matrix began to slacken off, the photo-thermal conversion efficiency returned to its original state. Reprinted with permission from ref. 44. Copyright 2014, American Chemical Society. (E) In vivo photo-thermal conversion imaging and tumor volume curves. Reprinted with permission from ref. 45. Copyright 2017, American Chemical Society. 
photo-thermal conversion efficiency. ${ }^{44}$ Due to the rupture of the cell membrane by multiple cycles of radiation, the photothermal efficiency returned to its original state (Fig. 2D). To further improve the accumulation of PTAs with the EPR effect, Jiang and coworkers used cyclopamine to disrupt the extracellular matrix of pancreatic ductal adenocarcinoma (PDA). ${ }^{45}$ As the tumor blood perfusion was improved, the erythrocyte membrane modified gold nanorods (MGNRs) showed a higher tumor accumulation (1.8-fold that of the control group). As a result, with the integrated advantages of tumor microenvironment regulation and long-circulation biomimetic MGNRs, outstanding in vivo photo-thermal and therapeutic effects were achieved (Fig. 2E).

\subsection{Leukocyte-based PTAs}

In addition to erythrocytes, white blood cells (WBCs) including granulocytes, monocytes, and lymphocytes have also received great attention. As the main participants in cellular immunity and humoral immunity, leukocytes can not only resist the invasion of bacteria, viruses, and other microorganisms, but also kill and inhibit tumors. ${ }^{57,58}$ Additionally, leukocytes exhibit deforming movement across the vascular barrier and migrate to the diseased region under the chemotaxis of inflammatory factors, when inflammation occurs. ${ }^{59}$ Thus, coating of leukocyte membranes might improve PTAs' penetration into the tumor site through active targeting and enhance the photothermal effect by accumulating PTAs. ${ }^{60}$

As the primary leukocytes, neutrophils (NEs) migrate to inflammatory sites rapidly. It is known that the explosion growth of tumors would cause chronic inflammation; thus the NEs will be constantly recruited to the tumor sites. ${ }^{61} \mathrm{Su}$ et al. developed a biomimetic nanoplatform (NG/BP-PEI-LY NPs) consisting of black phosphorus (BP), TGF- $\beta$, and the NE membrane. ${ }^{46}$ It is found that, by intravenous injection, the as-obtained NG/BP-PEI-LY NPs were gradually accumulated at the tumor site through the EPR effect and chemotaxis of few inflammatory factors (Fig. 3A). Upon NIR laser irradiation, the outstanding PTT and photo-dynamic therapy (PDT) effects stimulated acute inflammatory response, which in turn recruited much more NG/BP-PEI-LY NPs during the second administration $(30.9 \%$ improvement in the fluorescence intensities at the tumor site, Fig. 3B). As a result, the maximum temperature in the second round of irradiation increased by $3.9{ }^{\circ} \mathrm{C}$ (Fig. 3C). This treatment-induced self-amplification strategy showed a strong antitumor and anti-metastasis effect (Fig. 3D).

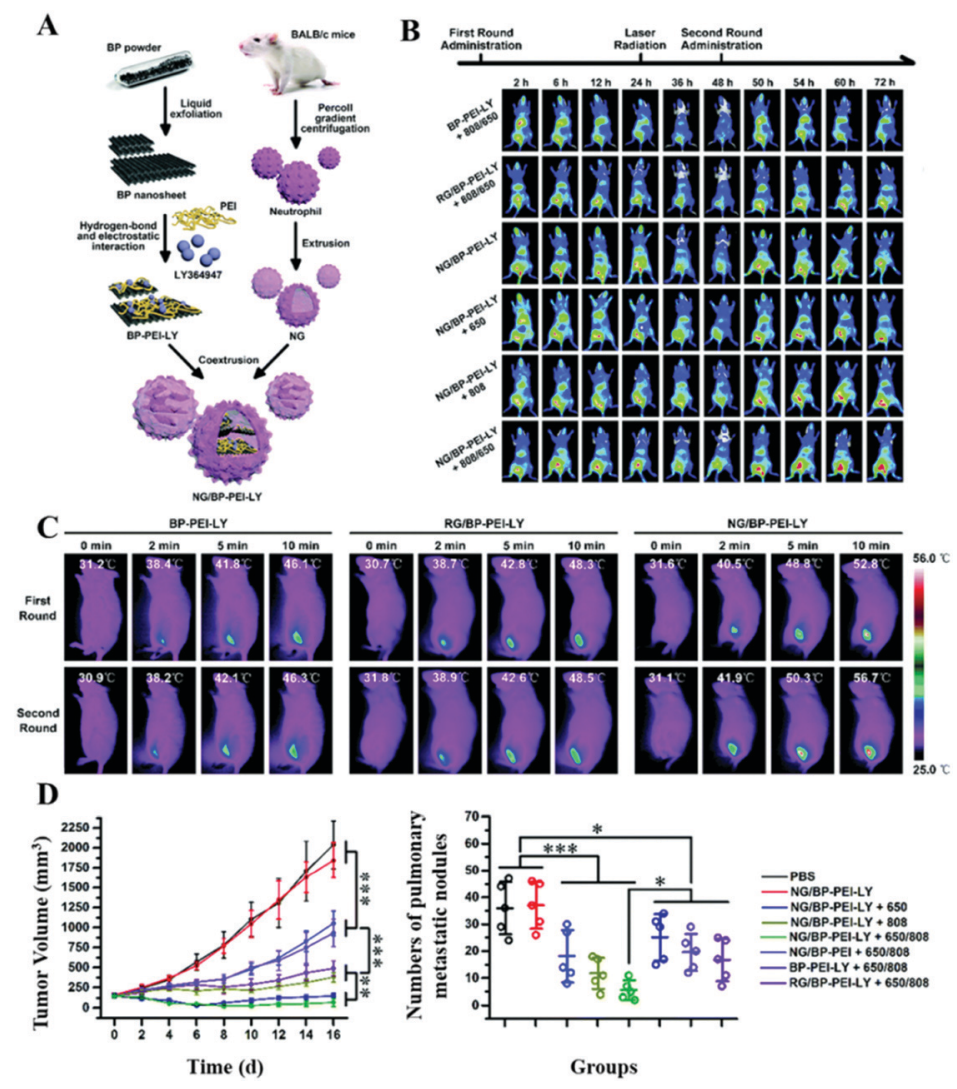

Fig. 3 (A) Schematic representations of the formation of NG/BP-PEI-LY NPs using black phosphorus (BP), TGF- $\beta$, and the NE membrane. (B) Fluorescence imaging and $(C)$ photo-thermal conversion imaging of 4T1 orthotopic transplantation tumor-bearing mice with different treatments. Once the NG/BP-PEI-LY NPs were intravenously injected into the tumor-bearing mice, there would be an obvious accumulation at the tumor site through the EPR effect and further chemotaxis of inflammatory factors caused by irradiation. (D) Tumor volume curves and quantitative analysis of pulmonary metastatic nodules in different groups. Reprinted with permission from ref. 46. Copyright 2020, Royal Society of Chemistry. 
In comparison with neutrophils, the stronger inflammationdirected chemotactic ability of macrophages can not only drive the accumulation of PTAs in chronic inflammatory tumor tissue but also swallow tumor cells directly. ${ }^{62}$ Li et al. used macrophages as bio-carriers to deliver gold nanorods (Au NRs) to tumor sites for PTT. $^{47}$ Via macrophage vehicles, the FITC labeled Au NRs could gradually penetrate the tumor mass and achieve an enhanced tumor coverage. However, the pure FITC-labeled Au NRs administered by intratumoral injection could hardly overcome the extracellular matrix to reach the tumor tissues (Fig. 4A). Nguyen et al. used RAW 264.7 macrophages to phagocytize small-sized gold nanorods and doxorubicin (DOX)-containing temperature-sensitive liposomes (DOX-LPs). ${ }^{48}$ The obtained AuNRs + DOX-LPs@ RAW showed a high penetration efficiency $(>10 \%)$ with 3D ellipsoidal tumors (4T1 cells) in vitro. In addition, the remarkable tumor targeting ability in vivo was verified by intravenously injecting DiD (fluorescent probe)-labeled AuNRs + DOX-LPs@ RAW into 4T1 tumor-bearing mice (Fig. 4B). In the following antitumor study, after the intratumoral injection of AuNRs + DOX-LPs@RAW, the temperature in the tumor site increased to $54.6{ }^{\circ} \mathrm{C}$ upon $808 \mathrm{~nm}$ laser irradiation $\left(1.5 \mathrm{~W} \mathrm{~cm}^{-2}\right.$ for $\left.5 \mathrm{~min}\right)$, which was higher than that in the AuNPs + DOX-LP treated mouse. Moreover, the heat accelerated release of DOX from the liposomes would further promote the elimination of tumor cells (Fig. 4C).
Immune leukocytes like $\mathrm{T}$ cells can alter targeting properties due to the variation of the microenvironment. The activated $\mathrm{T}$ cells can specifically recognize the major histocompatibility complex (MHC) on the surfaces of tumor cells through the $\mathrm{T}$ cell antigen receptor (TCR) on the membrane, exhibiting natural and high tumor affinity. ${ }^{63,64}$ Han et al. developed coreshell nanoparticles ( $\mathrm{N}_{3}$-TINPs) by translocating $\mathrm{N}_{3}$-modified natural $\mathrm{T}$ cell membranes on photosensitizer agents (hybrid of ICG and polylactic acid) (Fig. 4D). ${ }^{49}$ In addition to the retained immune recognition for tumor active targeting, the $\mathrm{N}_{3}$ group on $\mathrm{T}$ cells can specially bind to the bicyclo[6.1.0]nonyne (BCN) motif modified tumor cell membranes through bio-orthogonal chemistry reaction. Through a dual-targeting strategy, $\mathrm{N}_{3}$-TINPs aggregated in large numbers at the tumor site (monitored by fluorescence imaging with Raji cell-bearing NOD/SCID mice), which is 1.5 times higher than that with pure TINPs. The maximum temperature in the $\mathrm{N}_{3}$-TINP treated mice could reach $53{ }^{\circ} \mathrm{C}$ upon $808 \mathrm{~nm}$ laser irradiation $\left(0.8 \mathrm{~W} \mathrm{~cm}^{-2}, 5 \mathrm{~min}\right)$, resulting in the elimination of tumors after 16 days.

\subsection{Platelet based PTAs}

Platelets (PLTs), another natural component of blood, are produced by mature megakaryocytes. When blood vessels are damaged or ruptured, static platelets are stimulated to stretch
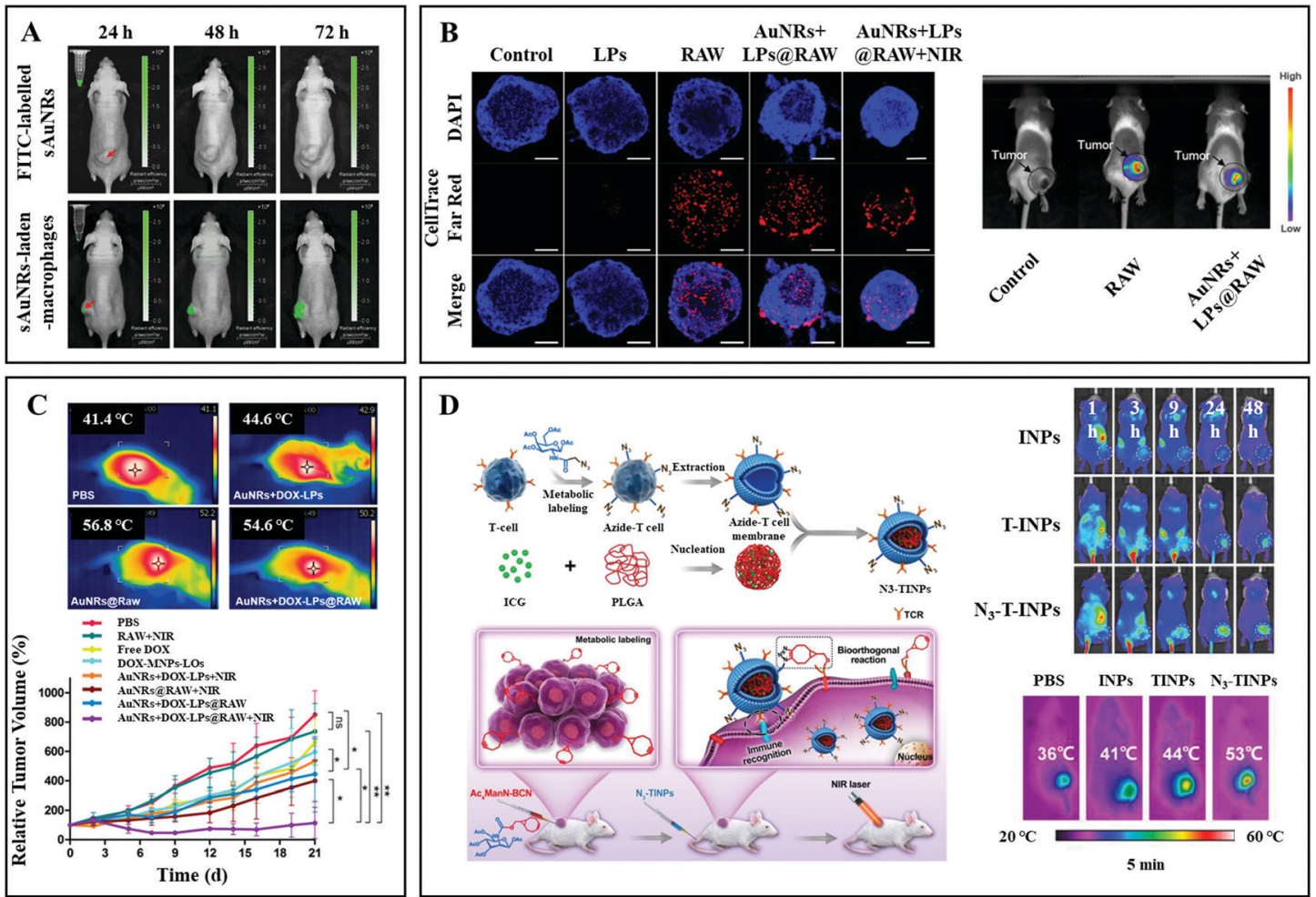

Fig. 4 (A) Fluorescence imaging of HepG2 tumor-bearing nude mice at $24 \mathrm{~h}, 48 \mathrm{~h}$, and $72 \mathrm{~h}$ after intratumoral injection of FITC labelled Au NRs (top) and macrophage carried FITC labelled Au NRs (bottom). Reprinted with permission from ref. 47. Copyright 2016, Elsevier. (B) In vitro and in vivo tumor (4T1) penetration ability tests of macrophages with and without NP payloads. (C) Photo-thermal conversion imaging and tumor volume curves of different treatment groups. Reprinted with permission from ref. 48. Copyright 2020, American Chemical Society. (D) Schematic illustration of the formation of $\mathrm{N}_{3}$-labeled T cell membrane coated NPs, and their antitumor application. Reprinted with permission from ref. 49. Copyright 2019, John Wiley \& Sons. 
out tentacles on their surfaces to trap plasma proteins and clotting factors. Thereafter, the platelets further release thrombin to induce the conversion of fibrinogen to fibrin, which net the red blood cells to form a solid haemostatic embolus, blocking the damaged wounds and vessels. ${ }^{65}$ Inspired by this, Wu et al. developed a biomimetic antitumor platform with PLT membranes, NIR laser responsive polypyrrole (PPy) NPs, and DOX. $^{50}$ Pure PLT membranes were developed by a repeated freeze-thaw process. Due to the immune evasion ability of the PLT membrane, the hybrid PTAs had a higher accumulation in a Huh7 orthotopic tumor, resulting in an obvious temperature increase (the maximum temperature in a PLT-PPy + laser mouse and a PLT-PPy-DOX + laser mouse was $50.3{ }^{\circ} \mathrm{C}$ and $51.2{ }^{\circ} \mathrm{C}$, respectively) (Fig. 5A). Consequently, the hepatocellular carcinoma cells were significantly eliminated by the combination of PTT and temperature controlled chemotherapy (Fig. 5B). Rao et al. directly used entire PLTs as biocarriers to deliver gold nanorods (AuNRs) for precise and enhanced tumor PTT. ${ }^{51}$ Through the electroporation process, the Au NRs were easily embedded into PLTs. Apart from their good biocompatibility and long circulation time in vivo, the administered PLT-Au NRs were continuously attracted to the tumor sites because of the applied localized $808 \mathrm{~nm}$ laser irradiation. In stark contrast to the pristine $\mathrm{Au}$ NR and PLT membrane-Au NP treatments, the experimental group exhibited an enhanced photo-thermal conversion effect (about $4{ }^{\circ} \mathrm{C}$ increase after each round of exposure, Fig. 5C) and tumor inhibition (Fig. 5D).

\subsection{Cancer cell based PTAs}

In stark contrast to leukocytes, cancer cells are not restricted by a variety of antigens, which might induce immunogenicity.
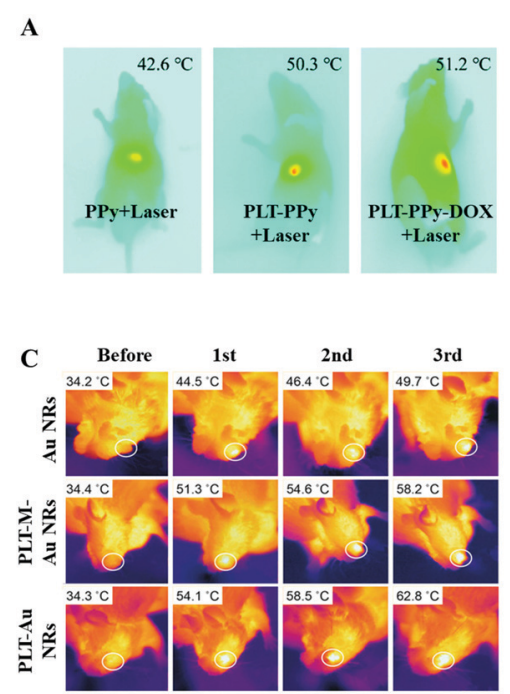
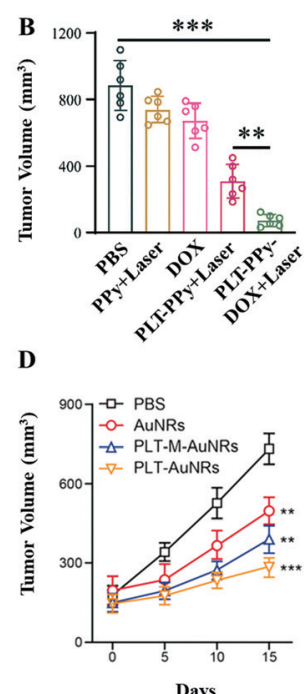

Fig. 5 (A) Infrared thermal images of Huh7 orthotopic tumor-bearing mice upon $808 \mathrm{~nm}$ laser irradiation $\left(2.5 \mathrm{~W} \mathrm{~cm}^{-2}\right.$ for $5 \mathrm{~min}$ ). (B) Tumor volume data. Reprinted with permission from ref. 50. Copyright 2020, Royal Society of Chemistry. (C) Infrared thermal images of CAL-27 tumorbearing mice upon $808 \mathrm{~nm}$ laser irradiation ( $2 \mathrm{~W} \mathrm{~cm}^{-2}$ for $5 \mathrm{~min}$ ) for different treatment periods. (D) Tumor volume curves. Reprinted with permission from ref. 51. Copyright 2018, John Wiley \& Sons.
The major histocompatibility complex (MHC) as an antigenpresenting molecule has extremely low expression on the membranes of cancer cells. Thus, cytotoxic $\mathrm{T}$ lymphocytes (CTLs) cannot recognize the cancer antigens, which will further facilitate immune escape. ${ }^{66}$ Additionally, cancer cell membrane-derived platforms with diameters of less than $200 \mathrm{~nm}$ have long residence times in circulation. This behavior increases the possibility to pass through tumor regions and permeate tumor tissues. It is reported that the over-expressed surface adhesion molecules and homologous adhesion domains on the membranes of cancer cells are responsible for multicellular aggregation formation, thus improving the retention rate of PTAs in the tumor tissues. ${ }^{67}$

Chen et al. developed a cancer cell membrane cloaked nanoparticle system (ICNPs), consisting of an ICG/polylactic acid (PLGA) self-assembly core and a cancer cell (MCF-7) membrane shell, which can synchronously recognize and eradicate tumors (Fig. 6A). ${ }^{52}$ Apart from the FL/PA dual-modal imaging and photo-thermal conversion abilities inherited from ICG, the resulting ICNPs with tumor-associated antigens adhere to the surfaces of solid tumors composed of MCF-7 (human breast cancer) cells more efficiently (3.1 fold compared to INPs and 4.75 fold compared to ICG). In addition, the ICNPs could not be easily intercepted by the liver and kidney due to the perfect camouflage, resulting in the increasing accumulation of PTAs. Consequently, significant photo-thermal conversion $\left(55.3{ }^{\circ} \mathrm{C}\right)$ and antitumor therapeutic effects were achieved upon NIR light irradiation in vivo. More recently, a biomimetic drug delivery system was designed by coating the $4 \mathrm{~T} 1$ cancer cell membrane on DOXloaded gold nanocages (Fig. 6B). ${ }^{53}$ The resulting CDAuNs could rapidly and selectively target the breast tumor sites. Upon NIR laser irradiation, the hyperthermia from PTT further induced DOX release. The excellent synergistic treatment strategy presented a tumor-suppressive rate of $98.9 \%$ in the original tumor and $98.5 \%$ in metastatic tumors, respectively.

\subsection{Bacteria-based PTAs}

Bacteria, as one of the most common microorganisms, have been proved to have antitumor effects. ${ }^{68}$ The non-toxicity of engineered bacteria can directly help $\mathrm{T}$ lymphocytes to recognize and kill tumor cells. ${ }^{69}$ In addition, salmonella in tumors could induce cell apoptosis through various mechanisms such as competition for nutrition, stimulation of an immune response, or release of toxins. Furthermore, owing to the anoxic environment of tumors, the accumulation of bacteria in tumors is $\sim 1000$ timest higher than that in normal organs. ${ }^{70}$ Therefore, bacteria are often used as vehicles to deliver therapeutic agents including chemical toxins, DNA, enzymes, and nanoparticles to solid tumor tissues. ${ }^{71}$ Chen et al. coated polydopamine on the surface of facultative anaerobe Salmonella VNP20009 to form pDA-VNP. ${ }^{54}$ The obtained bio-materials penetrated and grew in the avascular, hypoxic areas of deep tumors after intravenous administration. The local temperature in the center of tumors could reach $55.1{ }^{\circ} \mathrm{C}$ upon NIR laser irradiation (808 nm, $1.18 \mathrm{~W} \mathrm{~cm} \mathrm{~cm}^{-2}$ for $5 \mathrm{~min}$ ), which was sufficient for tumor PTT (Fig. 7A). In this process, pDA-VNP also stimulated 

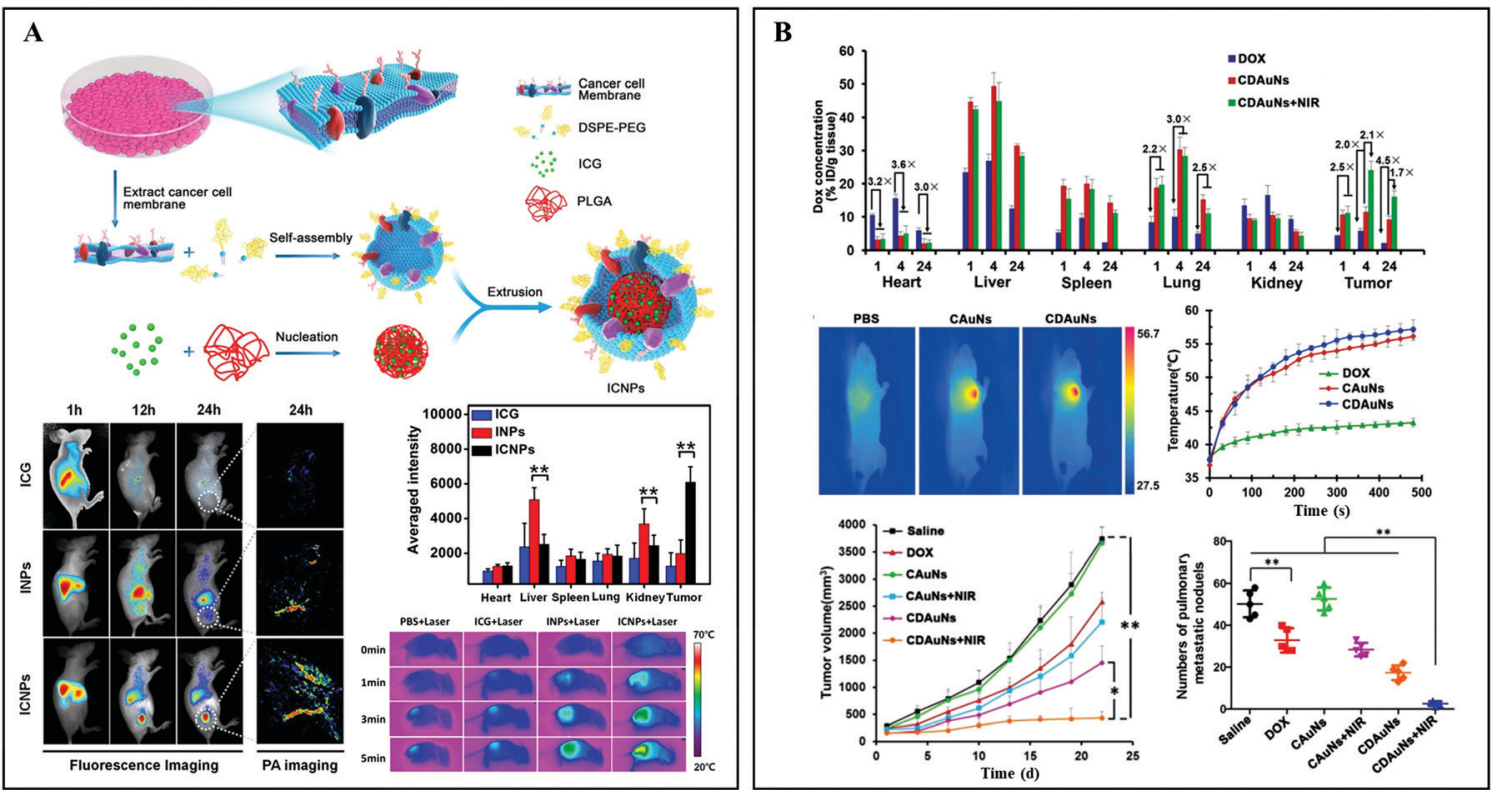

Fig. 6 (A) Schematic illustration of cancer cell membrane-derived bio-mimetic nanovehicles for specific tumor active imaging and photo-thermal therapy. Reprinted with permission from ref. 52. Copyright 2016. American Chemical Society. (B) 4 T1 cell membrane-cloaked Au nanocages for active tumor chemotherapy and photo-thermal therapy of the original tumor and metastatic tumors. Reprinted with permission from ref. 53. Copyright 2016, John Wiley \& Sons.

immune cells (natural killer cells, $\mathrm{CD}^{+} / \mathrm{CD}^{+}{ }^{+}$lymphocytes) to migrate to the tumor tissues and secrete IFN- $\gamma$ and TNF- $\alpha$, generating cellular and humoral immunity (Fig. 7B). Consequently, this synergistic treatment strategy eliminated all the cancer cells and no recurrence was observed (Fig. 7C).

\section{Protein-based PTAs}

As the most important functional biological macromolecule in the body, proteins are involved in all cell activities. Because of their special spatial structure, outstanding biocompatibility, and multifunctionality, protein-based PTAs have received tremendous attention. The types of proteins, sources, types of photosensitive agents, merits, dominant functions, and shortcomings are summarized and compared in Table 2.

\subsection{Natural or recombinant protein-based PTAs}

Owing to its good biocompatibility and availability, serum protein, a kind of globular protein, has been approved by the FDA for clinical use. ${ }^{81,82}$ Additionally, the natural hydrophobic domain in the center of serum protein promotes its use as a
A

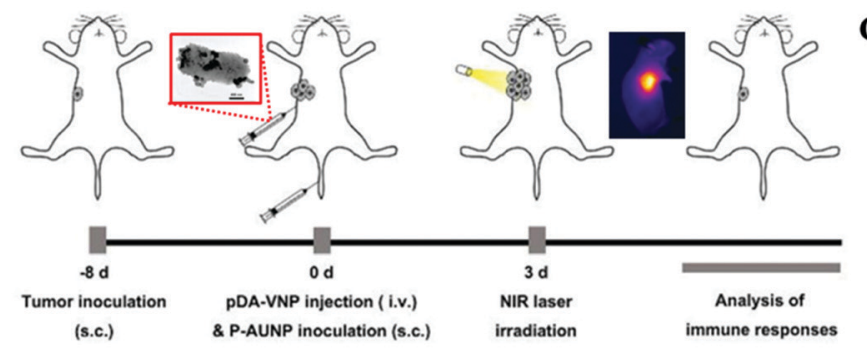

C

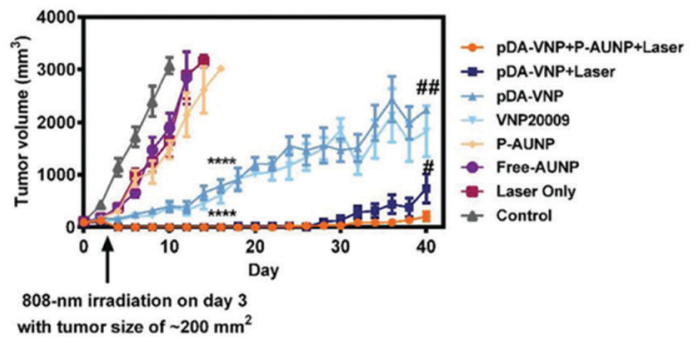

B
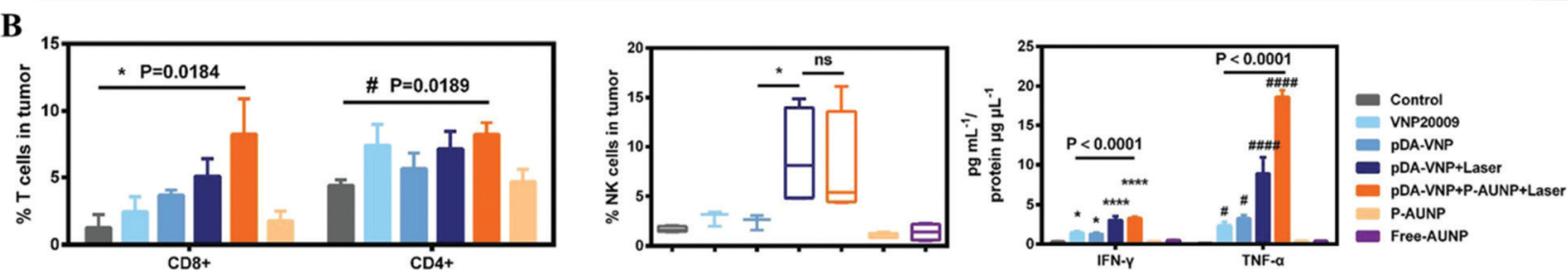

Fig. 7 (A) Schematic illustration of the utilization of polydopamine coated facultative anaerobe Salmonella VNP200094T1 for enhanced tumor photothermal therapy and PD-1 blockage induced immune therapy. (B) In vivo immune response including $\mathrm{CD} 8^{+} / \mathrm{CD} 4^{+} \mathrm{T}$ lymphocytes and antitumor factors. (C) Tumor volume curves. Reprinted with permission from ref. 54. Copyright 2019, John Wiley \& Sons. 
Table 2 Summary of the types of proteins, sources, types of photosensitive agents, merits, dominant functions, and shortcomings in the application in tumor PTT

\begin{tabular}{|c|c|c|c|c|}
\hline Type of protein & Source & $\begin{array}{l}\text { Photosensitive } \\
\text { agents }\end{array}$ & Merits and functions & Shortcomings \\
\hline $\begin{array}{l}\text { Bovine serum } \\
\text { albumin }^{72,73} \\
\text { Human serum } \\
\text { albumin }^{39,74}\end{array}$ & $\begin{array}{l}\text { From cow } \\
\text { blood } \\
\text { From human } \\
\text { blood }\end{array}$ & $\begin{array}{l}\text { ICG } \\
\text { Rhodamine } \\
\text { Black } \\
\text { phosphorus } \\
\mathrm{Ag}_{2} \mathrm{~S} \\
\text { nanoparticles }\end{array}$ & $\begin{array}{l}\text { Nanocarriers of small molecules; enhanced bio- } \\
\text { compatibility; synthesis template or vessel }\end{array}$ & $\begin{array}{l}\text { No active targeting abilities } \\
\text { towards solid tumors }\end{array}$ \\
\hline Ovalbumin $^{75}$ & Egg & ICG & Abundant source; immunotherapy & \\
\hline Silk fibroin ${ }^{76}$ & Silk & $\begin{array}{l}\text { Nano-graphene } \\
\text { oxide }\end{array}$ & Abundant source; component of hydrogel & Limited functional groups \\
\hline Collagen protein ${ }^{77}$ & Animal tissues & $\begin{array}{l}\text { Gold } \\
\text { nanoparticles }\end{array}$ & Abundant source; synthesis template & \\
\hline $\begin{array}{l}\text { Elastin-like } \\
\text { polypeptide }^{78}\end{array}$ & E. coli & $\begin{array}{l}\mathrm{Ag}_{3} \mathrm{AuS}_{2} \\
\text { nanoparticles }\end{array}$ & $\begin{array}{l}\text { Low immunogenicity; abundant functional groups; } \\
\text { component of hydrogel }\end{array}$ & $\begin{array}{l}\text { Constraints on synthesis } \\
\text { technology }\end{array}$ \\
\hline $\begin{array}{l}\text { Monoclonal antibody } \\
\text { (anti-EGFR) }\end{array}$ & $\begin{array}{l}\text { Hybridoma } \\
\text { technique }\end{array}$ & ICG & $\begin{array}{l}\text { Specific combination with the epidermal growth } \\
\text { factor receptor (EGFR) on cancer cells }\end{array}$ & $\begin{array}{l}\text { Expensive; not all tumor cells have } \\
\text { these antigens }\end{array}$ \\
\hline $\begin{array}{l}\text { Monoclonal antibody } \\
(\text { anti-P53) }\end{array}$ & & $\begin{array}{l}\text { Gold } \\
\text { nanoparticles }\end{array}$ & $\begin{array}{l}\text { Specific combination with mutant-type p } 53 \text { protein } \\
\text { over-expressing breast tumors }\end{array}$ & \\
\hline $\begin{array}{l}\text { Monoclonal antibody } \\
\text { (anti-VEGF) }^{80}\end{array}$ & & Graphene oxide & $\begin{array}{l}\text { Specific combination with the vascular endothelial } \\
\text { growth factor (VEGF) receptor on cancer cells }\end{array}$ & \\
\hline
\end{tabular}

carrier for water-insoluble organic dyes. ${ }^{72}$ Zhou et al. designed and synthesized novel PTAs, named RC-BSA, via the hybridization of rhodamine, hemicyanine derivatives, and bovine serum albumin (BSA). ${ }^{73}$ The as-obtained biomaterials with a diameter of about $135 \mathrm{~nm}$ showed strong NIR absorption in the NIR-I window. Upon $915 \mathrm{~nm}$ laser irradiation, the photo-thermal conversion efficiency could reach $28.7 \%$ (Fig. 8A). Through intratumoral administration and irradiation $\left(915 \mathrm{~nm}\right.$ laser, $1.0 \mathrm{~W} \mathrm{~cm}^{-2}$ for $10 \mathrm{~min}$ ), all the original tumors (4T1) disappeared within two days. More recently, Sheng et al. developed human serum albumin (HSA)-ICG hybrid nanoparticles (HSA-ICG NPs) by intermolecular disulfide conjugations. ${ }^{74}$ Compared to pristine ICG, the obtained HSA-ICG nanoparticles (about $75 \mathrm{~nm}$ ) presented a higher accumulation in tumor tissues $24 \mathrm{~h}$ post injection (the content of ICG is $28.8 \%$ dose per g, and the tumor-to-normal tissue ratio is $36.12 \pm 5.12$ ) (Fig. 8B). With the guidance of fluorescence and photo-acoustic dual-modal imaging, 808 laser irradiation $\left(0.8 \mathrm{~W} \mathrm{~cm}^{-2}\right.$ for $\left.5 \mathrm{~min}\right)$ was precisely applied to $4 \mathrm{~T} 1$ tumor-bearing mice $24 \mathrm{~h}$ after intravenous administration. As a result, all the cancer cells were eliminated by the PTT and PDT effects, and the survival rate remained at $100 \%$ for at least 50 days. In addition, serum protein can also be used as a synthesis template for inorganic materials. Yang et al. utilized HSA nanocages to fabricate $\mathrm{Ag}_{2} \mathrm{~S}$ NPs of various diameters in a precisely controlled manner (Fig. 8C). ${ }^{39}$ The prepared $\mathrm{Ag}_{2} \mathrm{~S}$ NPs exhibited obvious size dependent characteristics in NIR-II fluorescence, photo-thermal conversion efficiency, and residence time. In this regard, $\mathrm{Ag}_{2} \mathrm{~S}$ NPs with a diameter of $9.8 \mathrm{~nm}$ were used for in vivo antitumor study. All the 4T1 cancer cells were eliminated after intravenous administration $\left(50.0 \mu \mathrm{mol} \mathrm{kg}{ }^{-1}\right)$ upon $785 \mathrm{~nm}$ laser irradiation $\left(1.5 \mathrm{~W} \mathrm{~cm}^{-2}\right.$ for $\left.5 \mathrm{~min}\right)$. Furthermore, the natural globular protein can act as a nanovaccine to activate the immune system for tumor immunotherapy. Pan et al. constructed the simplest multifunctional nanovaccine by mixing ovalbumin (OVA) and ICG (Fig. 8D). ${ }^{75}$ The prepared OVA-ICG nanovaccine with $80.8 \%$ OVA and $19.2 \%$ ICG demonstrated remarkable NIR absorption and intense NIR fluorescent emission. In this study, the researchers found that the OVA could activate cellular immunity (secreting tumor necrosis factor- $\alpha$ ) and humoral immunity (secreting interleukin-6) simultaneously. Benefiting from the synergistic photo-thermal-immunotherapy, melanomabearing mice were well treated. In all, natural globular proteins are alternative candidates as nanocarriers for small molecule dyes and inorganic PTAs.

Apart from globular proteins, fibrous proteins, e.g., elastin, collagen, gelatin, and silk fibroin, with good biocompatibility are also widely utilized for fabricating hybrid photo-thermal materials. ${ }^{83} \mathrm{He}$ et al. developed a silk fibroin hydrogel by hybridizing upconversion nanoparticles (UCNPs) and nanographene oxide (SF/UCNP@NGO) (Fig. 9A). ${ }^{76}$ The UCNPs intratumorally injected with the hydrogel could be illuminated by $980 \mathrm{~nm}$ laser irradiation, which was used for tumor fluorescence imaging. Owing to the photo-thermal conversion ability of NGO, all the treated 4T1 tumors were completely suppressed upon $808 \mathrm{~nm}$ laser irradiation. In addition to the silk fibroin hydrogel, collagen protein hydrogels with inorganic anionic clusters were also applied to PTT (Fig. 9B). ${ }^{77}$ Taking advantage of the stability of the hydrogel in vivo, the loaded PTAs could be ensured at a therapeutic concentration through the PTT. However, the immunogenicity of proteins should be concerned when using such a large dose of natural proteins in vivo. ${ }^{84}$ In this regard, recombinant proteins or peptides with low immunogenicity have been designed and used for the preparation of photo-responsive hydrogels. ${ }^{85,86}$ Recently, Wei and coworkers developed a new type of injectable NIR lightresponsive hydrogel consisting of elastin-like polypeptide (ELP), chitosan, and $\mathrm{Au} / \mathrm{Ag}$ hybrid nanoparticles $\left(\mathrm{Ag}_{3} \mathrm{AuS}_{2} \mathrm{NPs}\right)$ via electrostatic interactions and forming amide bonds (Fig. 9C). ${ }^{78}$ The obtained hydrogel presented an outstanding photo-thermal conversion efficiency of $39.0 \%$, which was higher than that of the pristine nanoparticles (35.6\%). Beyond its photo-thermal ability, 

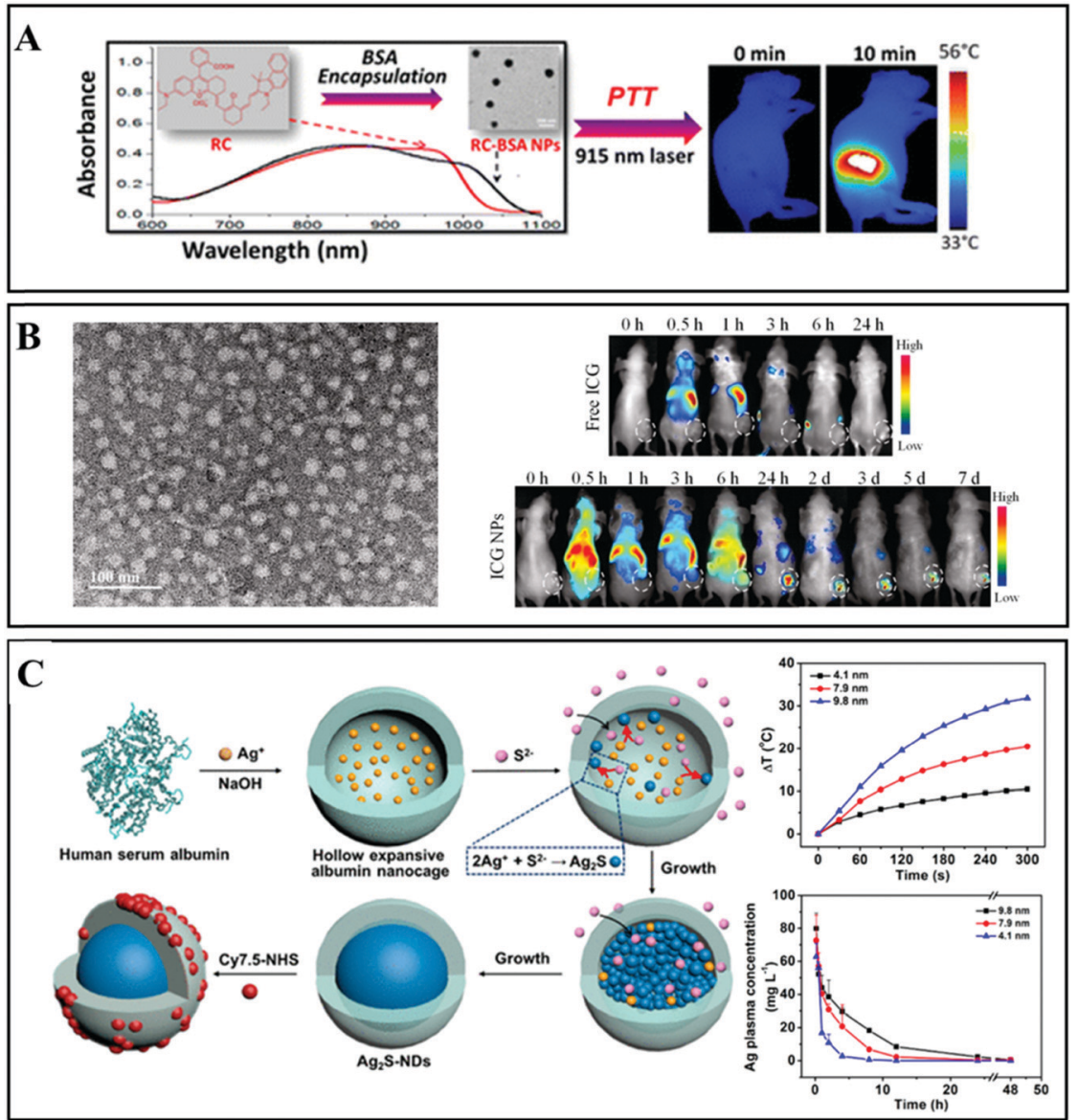
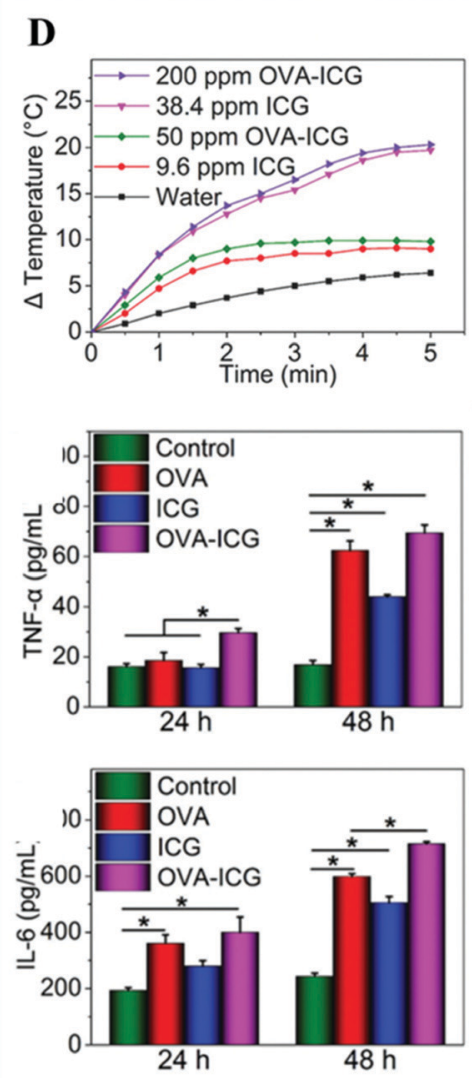

Fig. 8 (A) Schematic illustration of RC-BSA NPs for NIR laser induced PTT. Reprinted with permission from ref. 73. Copyright 2016, American Chemical Society. (B) Human serum albumin (HSA)-ICG hybrid nanoparticles for enhanced PTT. Reprinted with permission from ref. 74. Copyright 2014, American Chemical Society. (C) Schematic illustration of the preparation of $\mathrm{Ag}_{2} \mathrm{~S}$ NPs using albumin nanocages as the reaction template (left), and the size dependent photo-thermal conversion and distribution abilities. Reprinted with permission from ref. 39. Copyright 2017, American Chemical Society. (D) Photo-thermal conversion abilities of OVA-ICG hybrid NPs, and the accompanying immune response during the PTT in vivo. Reprinted with permission from ref. 75. Copyright 2018, John Wiley \& Sons.

the protein/polysaccharide-based hydrogel minimized the cytotoxicity of $\mathrm{Ag}_{3} \mathrm{AuS}_{2}$ NPs (73.6\% vs. 94.8\%), presenting high biocompatibility. In the antitumor study, through peritumoral administration and irradiation with an $808 \mathrm{~nm}$ laser (1.0 $\mathrm{W} \mathrm{cm}^{-2}$ ), the local temperature could rapidly exceed $50{ }^{\circ} \mathrm{C}$, which was high enough for tumor PTT. After 14 days of observation, tumors in tongue tumor-bearing mice were significantly inhibited after hydrogel treatment together with laser irradiation. The survival rate was remarkably improved due to the reversion of losing weight, suggesting the recovery of tongue functions.

\subsection{Functional protein-based PTAs}

Functional proteins play a variety of roles in immune regulation, hormonal regulation, and enzymatic reaction. Different from natural proteins, functional proteins are always used for nanoparticle surface functionalization rather than as carriers. Recently, active targeting based on specific ligands that target conjugated receptors on cancer cells has been investigated. Monoclonal antibodies (mAbs) prepared by hybridoma technology can only target the specific epitope on the corresponding cancer cells, which have been widely explored for immunotherapy and nanomedicine. Yu et al. coated a single layer of anti-epidermal growth factor receptor (anti-EGFR) biomolecules on polymer/salt/ICG aggregates (Fig. 10A). ${ }^{41}$ Taking advantage of the targeting properties of anti-EGFR, NPs could specifically bind to the surfaces of tumor cells that highly express EGFR (1483 cell line) (Fig. 10B). Apart from the inhibitory effect on tumors due to mAbs, an enhanced PTT effect was achieved owing to the additional accumulation of PTAs via active targeting. $\mathrm{Xu}$ et al. developed an ultra-small NIR responsive PTA (p53-PLGA@Au NPs), which can conjugate to the mutant-type p53 protein over-expressing breast tumors (MCF-7) and induce a site-specific PTT effect. ${ }^{79}$ Deng et al. utilized polyethylene glycol (PEG) as the "bridge" to conjugate anti-vascular endothelial growth factor (VEGF) antibodies on GO/HSA nanoparticles. ${ }^{80}$ In addition to the PTT effect, the release of thermally controlled Paclitaxel (PTX) further improved the antitumor effect. Consequently, the survival rate of experimental mice was still $100 \%$ after 100 days of observation. These results demonstrate 
A

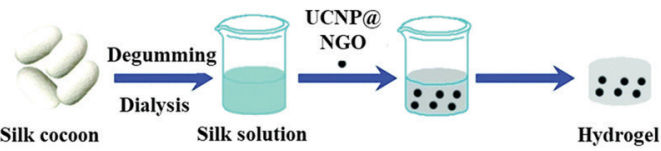

C

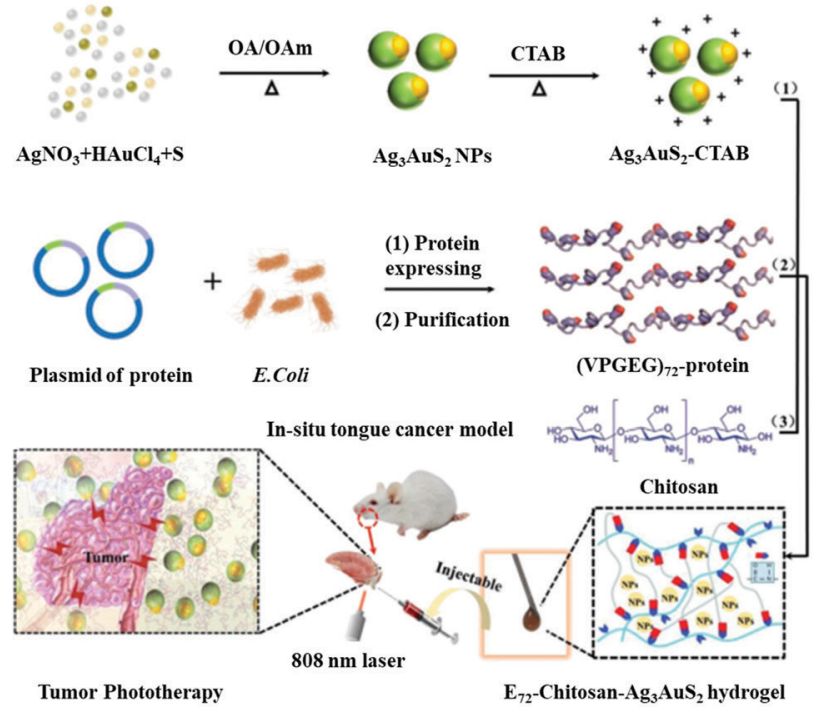

Fig. 9 Schematic illustration of the preparation and application of an injectable protein hybrid hydrogel. (A) Silk fibroin, upconversion nanoparticles, and nanographene oxide hybrid hydrogel. Reprinted with permission from ref. 76. Copyright 2019, Royal Society of Chemistry. (B) Inorganic anionic cluster loaded collagen protein hydrogels. Reprinted with permission from ref. 77. Copyright 2016, John Wiley \& Sons. (C) Schematic illustration of the preparation and application of an injectable protein hybrid hydrogel for in situ tongue cancer PTT. The main components of the hydrogel included a genetically engineered polypeptide (VPGEG) 72 , NIR light responsive $\mathrm{Ag}_{3} \mathrm{AuS}_{2}$ NPs, and chitosan. Reprinted with permission from ref. 78. Copyright 2021, John Wiley \& Sons.

that the conjugated mAbs can not only enhance the PTT effect by increasing the accumulation of PTAs via active targeting, but also induce an immune-mediated antitumor activity. ${ }^{87}$ Although mAbs demonstrated potential applications in the field of PTT, the high cost limits their widespread use in clinics.

\section{Nucleic acid-based PTAs}

The surface modifications of PTAs with nucleotides could improve the hydrophilia and biocompatibility of PTAs due to the abundant hydroxide radicals. Taking advantage of the hydrogen bonding between complementary bases, ${ }^{88}$ nucleotide sequences are often designed and synthesized to efficiently combine the over-expressed nucleotides in cancer cells, conducting specific functions, e.g., gene silencing, fluorescence signalling, drug delivery, and active targeting. ${ }^{84,89}$ The temperature responsive property of nucleotides could induce drug release.

Recently, Han et al. developed intelligent nanoconjugates comprising hairpin DNA (hPDNA) and UCNP@Au NPs (Fig. 11A). ${ }^{90}$ The double-helical framework of hpDNA provided
A

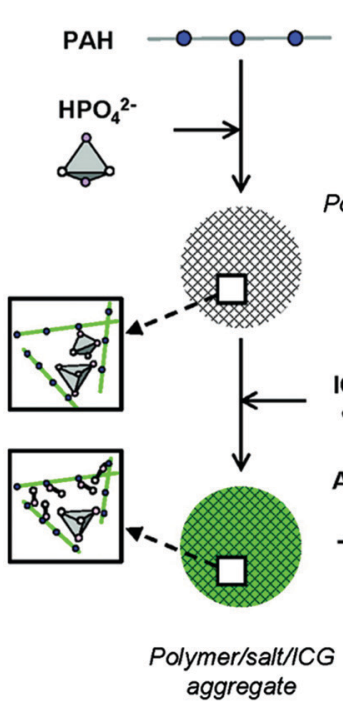

- Negatively charged

- Positively charged

○ Non charged

Polymer/salt aggregate

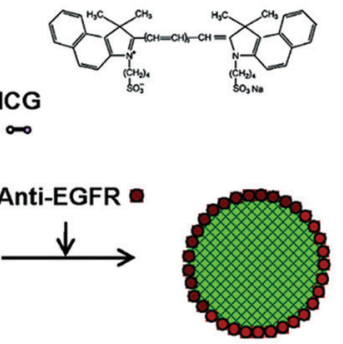

ICG-containing nanocapsules with antibody shell (ICG-NC's)
B
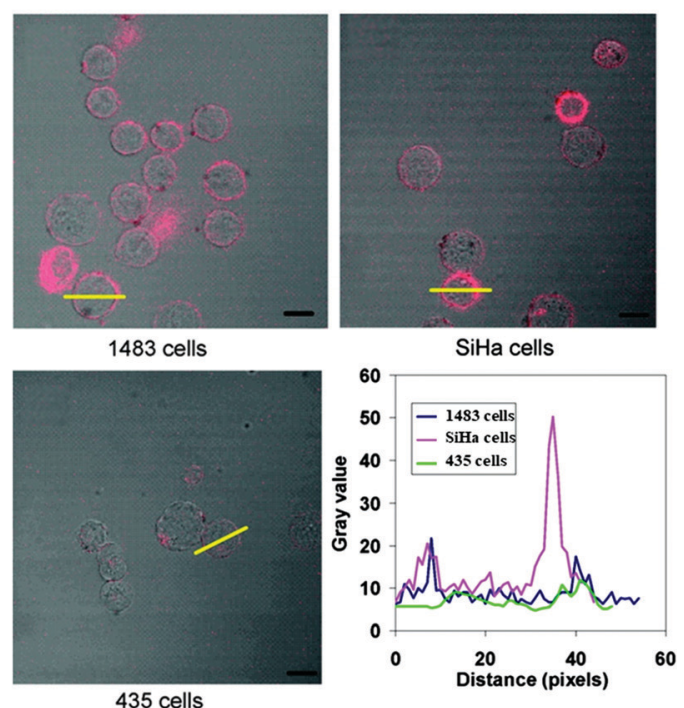

Fig. 10 (A) Schematic illustration of the preparation of anti-EGFR modified polymer/salt/ICG aggregates. (B) Presentation of tumor adhering abilities with fluorescence scanning. Reprinted with permission from ref. 41. Copyright 2010, American Chemical Society. 


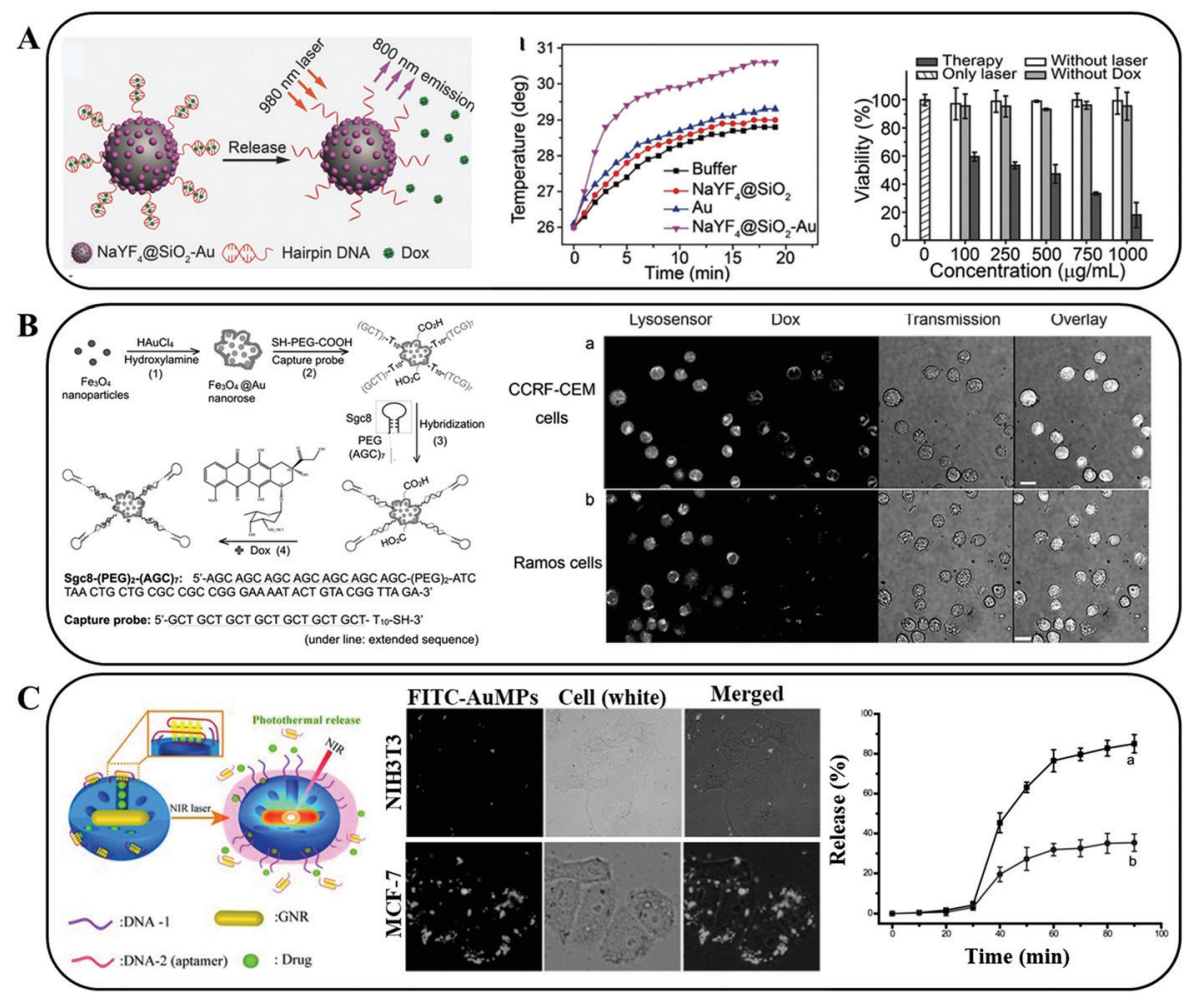

Fig. 11 (A) Schematic illustration of the NIR light triggered PTT effect and drug release. Reprinted with permission from ref. 90. Copyright 2017, John Wiley \& Sons. (B) Schematic illustration of the formation of sgc 8 aptamer modified gold-coated iron oxide $\left(\mathrm{Fe}_{3} \mathrm{O}_{4} @ \mathrm{Au}\right)$ nanoroses. The resulting hybrid NPs presented an active targeting ability towards CCRF-CEM leukaemia cells. Reprinted with permission from ref. 42. Copyright 2013 , John Wiley $\&$ Sons. (C) Schematic representation of the construction of aptamer covered AUMPs and the NIR triggered guest drug release with the binding aptamer dissociation. Reprinted with permission from ref. 92. Copyright 2012, John Wiley \& Sons.

sufficient space for small drug molecule insertion. Upon NIR laser irradiation, the hyperthermia generated from UCNP@Au NPs further induced the hpDNA to untwist and release DOX, resulting in a synergetic antitumor effect. Due to their spatial structure, the aptamers can specifically conjugate to their targets, e.g., proteins, cells, and even tissues. ${ }^{91}$ For instance, Li et al. conjugated sgc8 aptamers on prepared gold-coated iron oxide $\left(\mathrm{Fe}_{3} \mathrm{O}_{4} @ \mathrm{Au}\right)$ nanoroses (Fig. 11B). ${ }^{42}$ The resulting biomaterial demonstrated an active targeting ability towards CCRF-CEM leukemia cells. Based on the properties of dual molecular imaging, NIR absorption, and thermally-sensitive drug release, an observable killing of tumors was realized. In another research study, Yang et al. demonstrated a novel NIR light responsive antitumor drug nonvehicle based on mesoporous silica coated gold nanorods (AUMPs) and nucleolin targeting aptamers (Fig. 11C). ${ }^{92}$ A G-quadruplex DNA cap $(2.4 \mathrm{~nm})$ assembled with a 12-mer oligonucleotide and a 26-mer guanine-rich oligonucleotide DNA aptamer (AS1411) to gate the micropores $(2.5 \mathrm{~nm})$ and prevent the leakage of DOX. Under the guidance of AS1411, the nonvehicle achieved an active targeting to MCF-7 cells. When NIR laser irradiation was applied, the heat generated by Au NPs caused the duplex DNA to dehybridize, allowing the release of DOX.

Taking advantage of the specific pairings between complementary bases, nucleic acids are also used to prepare injectable hydrogels for loading PTAs. In our previous studies, we developed a DNA-inorganic hydrogel (DNA-UCNP-Au) by complexing salmon sperm DNA and cationic upconversion lanthanide-Au hybrid nanoparticles (UCNP-Au NPs) (Fig. 12A). ${ }^{93}$ Since the NIR-light responsive UCNP-Au NPs were confined in the network constructed of DNA strands, the light energy was converted to heat rapidly. In addition, the photothermal efficiency was improved from $32.44 \%$ to $42.7 \%$ in comparison to pristine PTAs (Fig. 12B). Tomoya Yata et al. used a hexapod-like structured DNA with $\mathrm{CpG}$ sequences to construct a hydrogel framework (Fig. 12C). The direct PTT effect due to the irradiated Au NPs accompanied by immunotherapy (by accelerating the production of tumor-associated IgG) further inhibited the proliferation of tumor cells. ${ }^{94}$

\section{Polysaccharide-based PTAs}

Natural polysaccharides, such as alginate, hyaluronic acid (HA), cellulose, and chitosan, have been widely investigated in medical fields due to their broad range of resources, good biocompatibility, non-toxicity, and biodegradability. ${ }^{95,96}$ Xing et al. demonstrated a facile and green method to synthesize cellulose and black phosphorus nanosheet (BPNS) hybrid hydrogels (Fig. 13A). The obtained hydrogels inheriting the excellent photo-thermal properties from BPNSs could effectively inhibit the proliferation of SMMC-7721 tumors via 

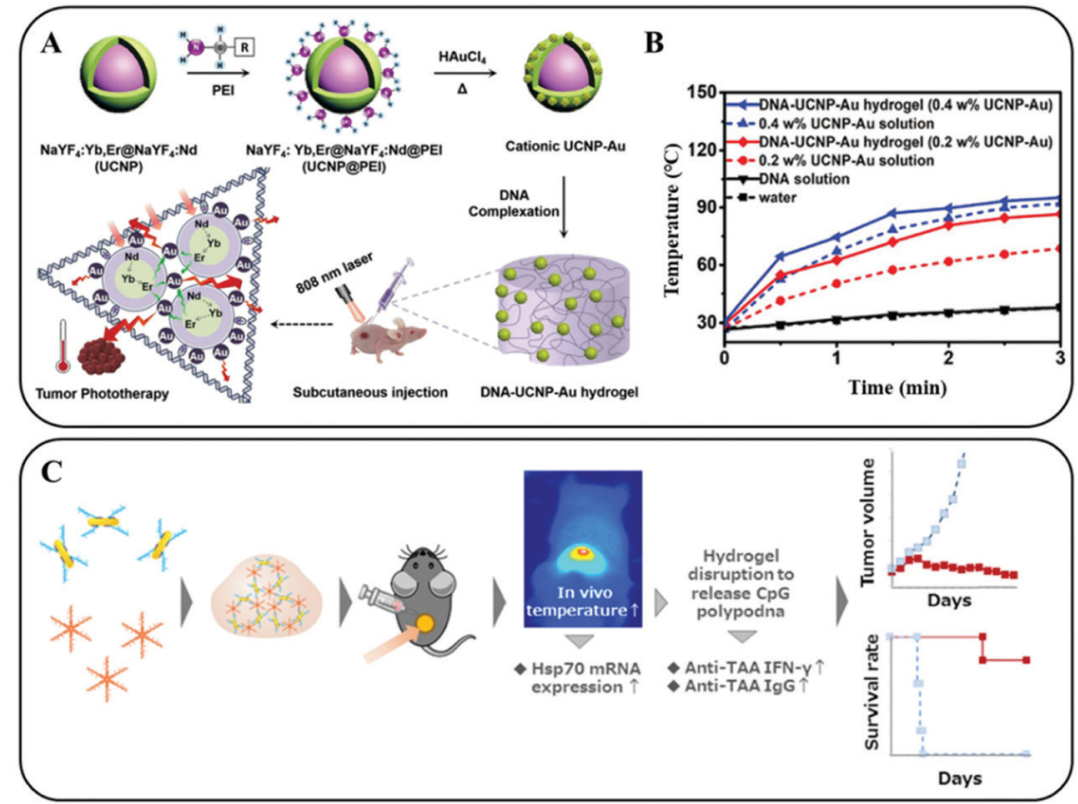

Fig. 12 (A) Schematic illustration of the preparation and application of an injectable NIR light responsive hydrogel for enhanced PTT of tumors. (B) Temperature curves of the irradiated hydrogels and solutions containing different concentrations of PTAs. Reprinted with permission from ref. 93. Copyright 2020, John Wiley \& Sons. (C) Schematic representation of the construction and photo-thermal/photoimmune therapy application of a hexapod-like structured DNA-CpG sequence based hydrogel. Reprinted with permission from ref. 94. Copyright 2017, Elsevier.

intratumoral injection upon $808 \mathrm{~nm}$ laser irradiation (1 $\mathrm{W} \mathrm{cm}^{-2}$ for $\left.5 \mathrm{~min}\right)$ (Fig. 13B). ${ }^{97}$ Chen et al. reported an injectable drug delivery and light triggered release system, consisting of azobenzene, $\alpha$-cyclodextrin-functionalized HA, and mesoporous silica nanoparticle (MSN) modified gold nanobipyramids (Au NBs) (Fig. 13C). Due to the high adhering ability of $\mathrm{HA}$ to the surfaces of $\mathrm{CD}_{4} 4^{+}$cancer cells, an enhanced accumulation of HA modified PTAs in the tumors was
A

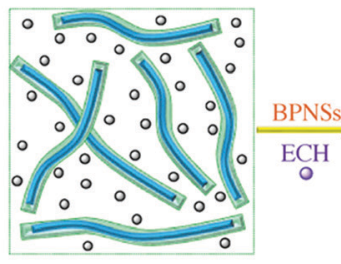

Cellulose Solution

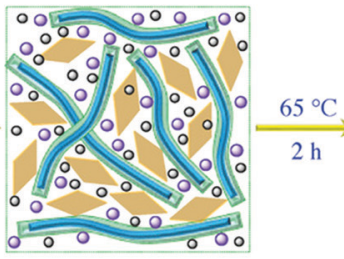

Cellulose/BPNSs/ECH Solution

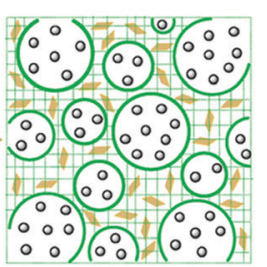

Cellulose/BPNSs Hydroge
B

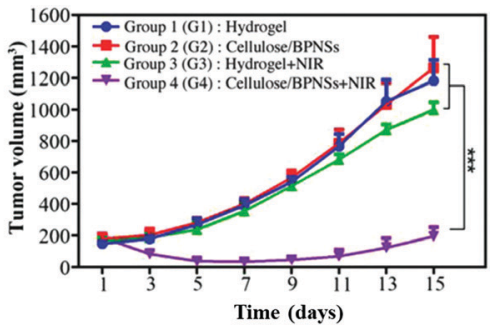

D

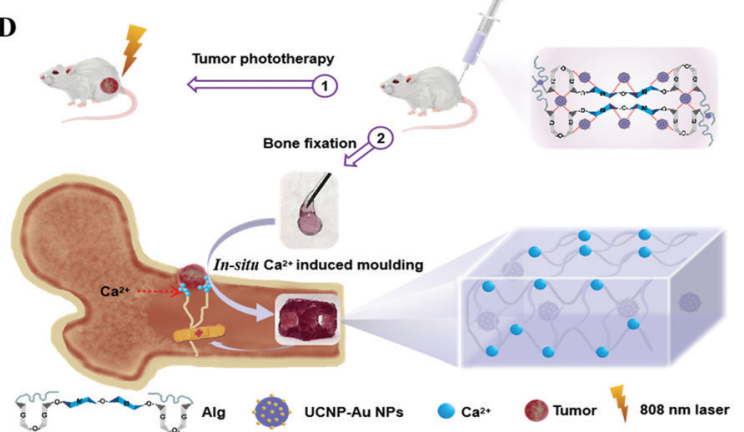

Fig. 13 (A) Schematic illustration of the preparation of an injectable cellulose and black phosphorus nanosheet (BPNS) composite hydrogel. (B) Tumor volume variations after different treatments. Reprinted with permission from ref. 97 . Copyright 2018, John Wiley \& Sons. (C) Schematic representation of the HA induced active drug delivery to cancer cells based on the specific adhesion to CD44 antigen. With NIR irradiation, the inserted PTAs were crosslinked due to the transformation of azobenzene. Reprinted with permission from ref. 98. Copyright 2016, American Chemical Society. (D) Schematic illustration of the UCNP-Au NPs and alginate conjugated hydrogel for tumor PTT and damaged bone repair. Reprinted with permission from ref. 12. Copyright 2021, John Wiley \& Sons. 
observed. When NIR laser irradiation was applied, the high temperature that originated from Au NBs not only accelerated the formation of an elastic hydrogel by crosslinking azobenzene with cyclodextrin but also had a good PTT effect. The slow release of DOX inhibited the recurrence of cancer cells. ${ }^{98}$ Similarly, calcium alginate based hydrogels have been tremendously explored as engineered materials for their matchable mechanical properties to soft tissues. One of our previous related studies demonstrated an injectable hydrogel containing NIR responsive UCNP-Au hybrid NPs and alginate with a high G/M ratio (Fig. 13D). ${ }^{12}$ Via peritumoral injection, the $\mathrm{Ca}^{2+}$ in the extracellular matrix facilitated the formation of a tough UCNP$\mathrm{Au}-\mathrm{Alg}-\mathrm{Ca}^{2+}$ hydrogel, isolating the tumors in space. All the cancer cells died from necrosis and apoptosis upon repeated $808 \mathrm{~nm}$ laser irradiation. Additionally, the damaged bone structure was stabilized and repaired using such hydrogels.

\section{Conclusions and perspectives}

Due to their minimally-invasive, direct, and precise characteristics of photo-thermal therapy, PTAs have been widely investigated in modern medicine. In stark contrast to organic molecules or inorganic nanoparticles, biomacromolecules exhibit excellent biocompatibility, structural controllability, and multi-functionality. Such behaviors can be exploited as functional primitives to manipulate the performance of next-generation PTAs. By taking advantage of simple coating, high photo-thermal effects, and low immunogenicity, biomacromolecule-based PTAs show promising application in cancer therapy. Moreover, the multi-functionality of biomacromolecule-based PTAs, such as imaging, targeting, and drug-loading, further broadens their applications in drug delivery and early diagnosis. Therefore, we summarized recent advances of biomacromolecule-based PTAs, such as cell-based PTAs, proteinbased PTAs, nucleotide-based PTAs, and polysaccharide-based PTAs. In addition, we also highlighted the functional design, structural control, and photo-thermal enhancement of such biomacromolecule-based PTAs.

Although a variety of biological macromolecule-based agents have been generated, there are still many challenges that still need to be addressed to take full advantage of biomacromolecules, especially in biomedical applications. On one hand, the tedious expression or synthetic routes of biomacromolecules limit their biomedical applications. ${ }^{99}$ On the other hand, it remains challenging to utilize currently reported biomacromoleculebased PTAs due to their poor structural stability. In addition, the photo-thermal efficiency of such materials should be further improved before their practical clinical application.

The design of stable biomacromolecule-based PTAs with high photo-thermal effects and biodegradability will greatly improve their photo-thermal behaviors in biomedical applications. To further promote the application of biomacromolecule-based PTAs, new synthetic biological techniques will be developed to achieve the mass production of biomacromolecules, e.g., functional proteins and nucleotides. The functionalization of biomacromolecules could also be integrated to improve the structural stability of PTAs in vivo. Due to the vigorous proliferation ability of cancer cells, it is difficult to completely eliminate them through one shot of irradiation in clinics. Therefore, researchers should pay more attention to immunotherapies with permanent anti-tumor effects. Using biomacromolecule-based PTAs as nanocarriers to deliver immunologic adjuvants or as factors to directly induce immune cascade reactions may have an enhanced antitumor effect. Additionally, the recognition and treatment of metastatic tumors and suspected lymph nodes through multifunctional PTAs should also be the focus of future studies. Overall, the development of biomacromolecule-based PTAs with various tailored properties and on-demand functionalities will open up new possibilities for nextgeneration biomedical materials and break through the current limitations in tumor treatment in clinics.

\section{Conflicts of interest}

There are no conflicts to declare.

\section{Acknowledgements}

This research was supported by the National Key R\&D Program of China (2020YFA0712102, 2020YFA0908900 and 2018YFA0902600), K. C. Wong Education Foundation (GJTD-2018-09), the National Natural Science Foundation of China (Grant No. 21877104, 21834007, and 22020102003), the Youth Innovation Promotion Association of CAS (Grant No. 2020228) and the Young Elite Scientists Sponsorship Program by CAST (Grant No. 2018QNRC001).

\section{Notes and references}

1 Y. Wang, X. Weng, L. Wang, M. Hao, Y. Li, L. Hou, Y. Liang, T. Wu, M. Yao and G. Lin, et al., J. Clin. Invest., 2018, 128, 5235-5250.

2 F. S. Ali, M. R. Hussain, C. Gutiérrez, P. Demireva, L. Y. Ballester, J.-J. Zhu, A. Blanco and Y. Esquenazi, Cancer Treat. Rev., 2018, 65, 33-40.

3 G. Wu, Y. Chen, Y. Wang, J. Yu, X. Lv, X. Ju, Z. Shi, L. Chen and Z. Chen, IEEE Trans. Med. Imaging, 2018, 37, 893-905.

4 J. Arita, T. Ishizawa, N. Akamatsu, J. Kaneko and K. Hasegawa, Ann. Oncol., 2019, 30, iv49.

5 M. Agn, P. Munck af Rosenschöld, O. Puonti, M. J. Lundemann, L. Mancini, A. Papadaki, S. Thust, J. Ashburner, I. Law and K. Van Leemput, Med. Image Anal., 2019, 54, 220-237.

6 I. A. Rodriguez-Brenes, A. V. Kurtova, C. Lin, Y.-C. Lee, J. Xiao, M. Mims, K. S. Chan and D. Wodarz, Cancer Res., 2017, 77, 2231.

7 C. D'Alterio, S. Scala, G. Sozzi, L. Roz and G. Bertolini, Semin. Cancer Biol., 2020, 60, 351-361.

8 F. Bednar and M. Pasca di Magliano, Cancer Discovery, 2020, 10, 762 .

9 I. Mokadem, W. P. M. Dijksterhuis, M. van Putten, L. Heuthorst, J. M. de Vos-Geelen, N. Haj Mohammad, G. A. P. Nieuwenhuijzen, H. W. M. van Laarhoven and R. H. A. Verhoeven, Gastric Cancer, 2019, 22, 1263-1273. 
10 S. Pandey, A. Talib, M. Mukeshchand Thakur, M. Shahnawaz Khan, M. L. Bhaisare, G. Gedda and H.F. Wu, J. Mater. Chem. B, 2016, 4, 3713-3720.

11 X. Wang, J. Zhang, J. Li, Y. Chen, Y. Chen, N. Kawazoe and G. Chen, J. Mater. Chem. B, 2019, 7, 3468-3469.

12 B. Liu, X. Gu, Q. Sun, S. Jiang, J. Sun, K. Liu, F. Wang and Y. Wei, Adv. Funct. Mater., 2021, 2010779.

13 D. Wang, B. Liu, Z. Quan, C. Li, Z. Hou, B. Xing and J. Lin, J. Mater. Chem. B, 2017, 5, 2209-2230.

14 W. Feng, X. Han, R. Wang, X. Gao, P. Hu, W. Yue, Y. Chen and J. Shi, Adv. Mater., 2019, 31, 1805919.

15 K. Zhao, J. Sun, F. Wang, A. Song, K. Liu and H. Zhang, ACS Appl. Bio Mater., 2020, 3, 3975-3986.

16 S. Wan, W. Cong, B. Shao, B. Wu, Q. He, Q. Chen, J. Shen, D. Chen, H.-G. Hu and F. Ye, et al., Nano Today, 2021, 38, 101115.

17 Q. Sun, J. Wu, L. Jin, L. Hong, F. Wang, Z. Mao and M. Wu, J. Mater. Chem. B, 2020, 8, 7253-7263.

18 M. Chang, M. Wang, Y. Chen, M. Shu, Y. Zhao, B. Ding, Z. Hou and J. Lin, Nanoscale, 2019, 11, 10129-10136.

19 W. Miao, G. Shim, S. Lee and Y.-K. Oh, Biomaterials, 2014, 35, 4058-4065.

20 W. Tao, X. Ji, X. Xu, M. A. Islam, Z. Li, S. Chen, P. E. Saw, H. Zhang, Z. Bharwani and Z. Guo, et al., Angew. Chem., Int. Ed., 2017, 56, 11896-11900.

21 J. Xie, T. Fan, J. H. Kim, Y. Xu, Y. Wang, W. Liang, L. Qiao, Z. Wu, Q. Liu and W. Hu, et al., Adv. Funct. Mater., 2020, 30, 2003891.

22 N. Fernandes, C. F. Rodrigues, A. F. Moreira and I. J. Correia, Biomater. Sci., 2020, 8, 2990-3020.

23 B. Shao, S. Wan, C. Yang, J. Shen, Y. Li, H. You, D. Chen, C. Fan, K. Liu and H. Zhang, Angew. Chem., Int. Ed., 2020, 59, 18213-18217.

24 S. Wan, F. Cui, B. Li, K. Zhao, H. He, Y. Zhang, J. Liu, L. Zhang and K. Liu, ACS Appl. Nano Mater., 2020, 3, 9433-9439.

25 L. Li, X. Han, M. Wang, C. Li, T. Jia and X. Zhao, Chem. Eng. J., 2021, 417, 128844.

26 Y. Zhang, K. Zhao, H. He, S. Wan, A. F. Martins, L. Zhang and K. Liu, Dalton Trans., 2021, 50, 2014-2017.

27 H. Zhu, P. Cheng, P. Chen and K. Pu, Biomater. Sci., 2018, 6, 746-765.

28 H. He, K. Zhao, L. Xiao, Y. Zhang, Y. Cheng, S. Wan, S. Chen, L. Zhang, X. Zhou and K. Liu, et al., Angew. Chem., Int. Ed., 2019, 58, 18286-18289.

29 O. Hanpanich and A. Maruyama, Biomaterials, 2020, 254, 120150.

30 C. Ma, B. Li, B. Shao, B. Wu, D. Chen, J. Su, H. Zhang and K. Liu, Angew. Chem., Int. Ed., 2020, 59, 21481-21487.

31 Y. Li, J. Li, J. Sun, H. He, B. Li, C. Ma, K. Liu and H. Zhang, Angew. Chem., Int. Ed., 2020, 59, 8148-8152.

32 J. Li, B. Li, J. Sun, C. Ma, S. Wan, Y. Li, R. Göstl, A. Herrmann, K. Liu and H. Zhang, Adv. Mater., 2020, 32, 2000964.

33 P. A. Boriack-Sjodin, S. Ribich and R. A. Copeland, Nat. Rev. Drug Discovery, 2018, 17, 435-453.
34 C. Ma, J. Su, Y. Sun, Y. Feng, N. Shen, B. Li, Y. Liang, X. Yang, H. Wu and H. Zhang, et al., Angew. Chem., Int. Ed., 2019, 58, 18703-18709.

35 C. Ma, B. Li, J. Zhang, Y. Sun, J. Li, H. Zhou, J. Shen, R. Gu, J. Qian and C. Fan, et al., Adv. Mater., 2021, 2100098.

36 Y. Yang, G. Mao, X. Ji and Z. He, J. Mater. Chem. B, 2020, 8, 9-17.

37 S. K. Burley, C. Bhikadiya, C. Bi, S. Bittrich, L. Chen, G. V. Crichlow, C. H. Christie, K. Dalenberg, L. Di Costanzo and J. M. Duarte, et al., Nucleic Acids Res., 2021, 49, D437-D451.

38 C. Ma, J. Su, B. Li, A. Herrmann, H. Zhang and K. Liu, Adv. Mater., 2020, 32, 1907697.

39 T. Yang, Y. a. Tang, L. Liu, X. Lv, Q. Wang, H. Ke, Y. Deng, H. Yang, X. Yang and G. Liu, et al., ACS Nano, 2017, 11, 1848-1857.

40 Y. Du, Q. Jiang, N. Beziere, L. Song, Q. Zhang, D. Peng, C. Chi, X. Yang, H. Guo and G. Diot, et al., Adv. Mater., 2016, 28, 10000-10007.

41 J. Yu, D. Javier, M. A. Yaseen, N. Nitin, R. Richards-Kortum, B. Anvari and M. S. Wong, J. Am. Chem. Soc., 2010, 132, 1929-1938.

42 C. Li, T. Chen, I. Ocsoy, G. Zhu, E. Yasun, M. You, C. Wu, J. Zheng, E. Song and C. Z. Huang, et al., Adv. Funct. Mater., 2014, 24, 1772-1780.

43 Q. Jiang, Z. Luo, Y. Men, P. Yang, H. Peng, R. Guo, Y. Tian, Z. Pang and W. Yang, Biomaterials, 2017, 143, 29-45.

44 J.-G. Piao, L. Wang, F. Gao, Y.-Z. You, Y. Xiong and L. Yang, ACS Nano, 2014, 8, 10414-10425.

45 T. Jiang, B. Zhang, S. Shen, Y. Tuo, Z. Luo, Y. Hu, Z. Pang and X. Jiang, ACS Appl. Mater. Interfaces, 2017, 9, 31497-31508.

46 Y. Su, T. Wang, Y. Su, M. Li, J. Zhou, W. Zhang and W. Wang, Mater. Horiz., 2020, 7, 574-585.

47 Z. Li, H. Huang, S. Tang, Y. Li, X.-F. Yu, H. Wang, P. Li, Z. Sun, H. Zhang and C. Liu, et al., Biomaterials, 2016, 74, 144-154.

48 V. D. Nguyen, H.-K. Min, D.-H. Kim, C.-S. Kim, J. Han, J.-O. Park and E. Choi, ACS Appl. Mater. Interfaces, 2020, 12, 10130-10141.

49 Y. Han, H. Pan, W. Li, Z. Chen, A. Ma, T. Yin, R. Liang, F. Chen, Y. Ma and Y. Jin, et al., Adv. Sci., 2019, 6, 1900251.

50 L. Wu, W. Xie, H.-M. Zan, Z. Liu, G. Wang, Y. Wang, W. Liu and W. Dong, J. Mater. Chem. B, 2020, 8, 4648-4659.

51 L. Rao, L.-L. Bu, L. Ma, W. Wang, H. Liu, D. Wan, J.-F. Liu, A. Li, S.-S. Guo and L. Zhang, et al., Angew. Chem., Int. Ed., 2018, 57, 986-991.

52 Z. Chen, P. Zhao, Z. Luo, M. Zheng, H. Tian, P. Gong, G. Gao, H. Pan, L. Liu and A. Ma, et al., ACS Nano, 2016, 10, 10049-10057.

53 H. Sun, J. Su, Q. Meng, Q. Yin, L. Chen, W. Gu, Z. Zhang, H. Yu, P. Zhang and S. Wang, et al., Adv. Funct. Mater., 2017, 27, 1604300.

54 W. Chen, Z. Guo, Y. Zhu, N. Qiao, Z. Zhang and X. Sun, Adv. Funct. Mater., 2020, 30, 1906623.

55 C.-M. J. Hu, R. H. Fang and L. Zhang, Adv. Healthcare Mater., 2012, 1, 537-547. 
56 S. Aryal, C.-M. J. Hu, R. H. Fang, D. Dehaini, C. Carpenter, D.-E. Zhang and L. Zhang, Nanomedicine, 2013, 8, 1271-1280.

57 C. Ju, Y. Wen, L. Zhang, Q. Wang, L. Xue, J. Shen and C. Zhang, Small, 2019, 15, 1804191.

58 S. Altmeier, A. Toska, F. Sparber, A. Teijeira, C. Halin and S. LeibundGut-Landmann, PLoS Pathog., 2016, 12, e1005882.

59 D. Chu, X. Dong, X. Shi, C. Zhang and Z. Wang, Adv. Mater., 2018, 30, 1706245.

60 P. Dash, A. M. Piras and M. Dash, J. Controlled Release, 2020, 327, 546-570.

61 E. Kolaczkowska and P. Kubes, Nat. Rev. Immunol., 2013, 13, 159-175.

62 A. C. Anselmo and S. Mitragotri, J. Controlled Release, 2014, 190, 531-541.

63 P. G. Coulie, B. J. Van den Eynde, P. van der Bruggen and T. Boon, Nat. Rev. Cancer, 2014, 14, 135-146.

64 T. Kang, Q. Zhu, D. Wei, J. Feng, J. Yao, T. Jiang, Q. Song, X. Wei, H. Chen and X. Gao, et al., ACS Nano, 2017, 11, 1397-1411.

65 C. E. Hansen, Y. Qiu, O. J. T. McCarty and W. A. Lam, Annu. Rev. Biomed. Eng., 2018, 20, 253-275.

66 D. Hanahan and R. A. Weinberg, Cell, 2011, 144, 646-674.

67 M. Zheng, C. Yue, Y. Ma, P. Gong, P. Zhao, C. Zheng, Z. Sheng, P. Zhang, Z. Wang and L. Cai, ACS Nano, 2013, 7, 2056-2067.

68 D. S. Leventhal, A. Sokolovska, N. Li, C. Plescia, S. A. Kolodziej, C. W. Gallant, R. Christmas, J.-R. Gao, M. J. James and A. Abin-Fuentes, et al., Nat. Commun., 2020, 11, 2739.

69 S. T. T. Schetters, W. S. P. Jong, S. K. Horrevorts, L. J. W. Kruijssen, S. Engels, D. Stolk, M. H. DalekeSchermerhorn, J. Garcia-Vallejo, D. Houben and W. W. J. Unger, et al., Acta Biomater., 2019, 91, 248-257.

70 V. H. Nguyen and J.-J. Min, Nucl. Med. Mol. Imaging, 2017, 51, 118-126.

71 B. F.-L. Sieow, K. S. Wun, W. P. Yong, I. Y. Hwang and M. W. Chang, Trends Cancer, 2021, 7, 447-464.

72 F. An, Z. Yang, M. Zheng, T. Mei, G. Deng, P. Guo, Y. Li and R. Sheng, J. Nanobiotechnol., 2020, 18, 49.

73 B. Zhou, Y. Li, G. Niu, M. Lan, Q. Jia and Q. Liang, ACS Appl. Mater. Interfaces, 2016, 8, 29899-29905.

74 Z. Sheng, D. Hu, M. Zheng, P. Zhao, H. Liu, D. Gao, P. Gong, G. Gao, P. Zhang and Y. Ma, et al., ACS Nano, 2014, 8, 12310-12322.

75 J. Pan, Y. Wang, C. Zhang, X. Wang, H. Wang, J. Wang, Y. Yuan, X. Wang, X. Zhang and C. Yu, et al., Adv. Mater., 2018, 30, 1704408.

76 W. He, P. Li, Y. Zhu, M. Liu, X. Huang and H. Qi, New J. Chem., 2019, 43, 2213-2219.

77 R. Xing, K. Liu, T. Jiao, N. Zhang, K. Ma, R. Zhang, Q. Zou, G. Ma and X. Yan, Adv. Mater., 2016, 28, 3669-3676.
78 J. Su, S. Lu, S. Jiang, B. Li, B. Liu, Q. Sun, J. Li, F. Wang and Y. Wei, Adv. Mater., 2021, 2100619.

79 L. Xu, C. Wan, J. Du, H. Li, X. Liu, H. Yang and F. Li, J. Biomater. Sci., Polym. Ed., 2017, 28, 415-430.

80 W. Deng, J. Qiu, S. Wang, Z. Yuan, Y. Jia, H. Tan, J. Lu and R. Zheng, Int. J. Nanomed., 2018, 13, 439-453.

81 J. Zhang, J. Sun, B. Li, C. Yang, J. Shen, N. Wang, R. Gu, D. Wang, D. Chen and $\mathrm{H}$. $\mathrm{Hu}$, et al., Small, 2020, 16, 1907598.

82 H. He, C. Yang, F. Wang, Z. Wei, J. Shen, D. Chen, C. Fan, H. Zhang and K. Liu, Angew. Chem., Int. Ed., 2020, 59, 4344-4348.

83 Z. Du, K. Yan, Y. Cao, Y. Li, Y. Yao and G. Yang, Mater. Sci. Eng., C, 2020, 117, 111340.

84 Q. Hu, H. Li, L. Wang, H. Gu and C. Fan, Chem. Rev., 2019, 119, 6459-6506.

85 S. Wang, B. Li, H. Zhang, J. Chen, X. Sun, J. Xu, T. Ren, Y. Zhang, C. Ma and W. Guo, et al., Angew. Chem., Int. Ed., 2021, 60, 11252.

86 L. Xiao, Z. Wang, Y. Sun, B. Li, B. Wu, C. Ma, V. S. Petrovskii, X. Gu, D. Chen and I. I. Potemkin, et al., Angew. Chem., Int. Ed., 2021, 60, 12082.

87 F. Claret and T. Vu, Front. Oncol., 2012, 2, 62.

88 S. Hoshika, I. Singh, C. Switzer, R. W. Molt, N. A. Leal, M.-J. Kim, M.-S. Kim, H.-J. Kim, M. M. Georgiadis and S. A. Benner, J. Am. Chem. Soc., 2018, 140, 11655-11660.

89 C. Husser, N. Dentz and M. Ryckelynck, Small Structures, 2021, 2000132.

90 S. Han, A. Samanta, X. Xie, L. Huang, J. Peng, S. J. Park, D. B. L. Teh, Y. Choi, Y.-T. Chang and A. H. All, et al., Adv. Mater., 2017, 29, 1700244.

91 X. Wang, X. Sun, H. He, H. Yang, J. Lao, Y. Song, Y. Xia, H. Xu, X. Zhang and F. Huang, J. Mater. Chem. B, 2015, 3, 3583-3590.

92 X. Yang, X. Liu, Z. Liu, F. Pu, J. Ren and X. Qu, Adv. Mater., 2012, 24, 2890-2895.

93 B. Liu, J. Sun, J. Zhu, B. Li, C. Ma, X. Gu, K. Liu, H. Zhang, F. Wang and J. Su, et al., Adv. Mater., 2020, 32, 2004460.

94 T. Yata, Y. Takahashi, M. Tan, H. Nakatsuji, S. Ohtsuki, T. Murakami, H. Imahori, Y. Umeki, T. Shiomi and Y. Takakura, et al., Biomaterials, 2017, 146, 136-145.

95 V. V. T. Padil, S. Wacławek, M. Černík and R. S. Varma, Biotechnol. Adv., 2018, 36, 1984-2016.

96 L. Li, N. Wang, X. Jin, R. Deng, S. Nie, L. Sun, Q. Wu, Y. Wei and C. Gong, Biomaterials, 2014, 35, 3903-3917.

97 C. Xing, S. Chen, M. Qiu, X. Liang, Q. Liu, Q. Zou, Z. Li, Z. Xie, D. Wang and B. Dong, et al., Adv. Healthcare Mater., 2018, 7, 1701510.

98 X. Chen, Z. Liu, S. G. Parker, X. Zhang, J. J. Gooding, Y. Ru, Y. Liu and Y. Zhou, ACS Appl. Mater. Interfaces, 2016, 8, 15857-15863.

99 S. Liu and L. G. Papageorgiou, Engineering, 2019, 5, 1077-1092. 